\title{
Multiple Periodic Solutions to a Kind of Lotka-Volterra Food-Chain System with Delays and Impulses on Time Scales
}

\author{
Kaihong Zhao, Liang Ding, and Fengzao Yang \\ Department of Applied Mathematics, Kunming University of Science and Technology, Yunnan, \\ Kunming 650093, China \\ Correspondence should be addressed to Kaihong Zhao, zhaokaihongs@126.com
}

Received 10 October 2012; Accepted 10 November 2012

Academic Editors: I. K. Argyros and H. T. Yau

Copyright (c) 2012 Kaihong Zhao et al. This is an open access article distributed under the Creative Commons Attribution License, which permits unrestricted use, distribution, and reproduction in any medium, provided the original work is properly cited.

By using Mawhin's continuation theorem of coincidence degree theory and some skills of inequalities, we establish the existence of at least $2^{n}$ periodic solutions for a kind of $n$-species Lotka-Volterra food-chain system with delays and impulses on time scales. One example is given to illustrate the effectiveness of our results.

\section{Introduction}

The food-chain phenomenon is universal and interesting in ecosystem. It is one of important methods to investigate this phenomenon by establishing the model of differential equations. The importance of studying the dynamics of food chain was pointed by Rosenzweig. In his famous paper on the paradox of enrichment [1], he wrote "Man must be very careful in attempting to enrich an ecosystem in order to increase its food yield. There is a real chance that such activity may result in decimation of the food species that are wanted in greater abundance." Hereafter, many scholars investigated various kinds of food-chain systems (see [2-9]).

In [10], Li and Zhao have studied the existence of multiple positive periodic solutions for the following Lotka-Volterra food-chain system:

$$
\dot{x}_{1}(t)=x_{1}(t)\left(a_{1}(t)-b_{1}(t) x_{1}(t)-c_{12}(t) x_{2}(t)\right)-h_{1}(t),
$$




$$
\begin{aligned}
\dot{x}_{i}(t)= & x_{i}(t)\left(-d_{i}(t)-b_{i}(t) x_{i}(t)+c_{i, i-1}(t) x_{i-1}(t)-c_{i, i+1}(t) x_{i+1}(t)\right) \\
& \quad-h_{i}(t), \quad 2 \leq i \leq n-1 \\
& \vdots \\
\dot{x}_{n}(t)= & x_{n}(t)\left(-d_{n}(t)-b_{n}(t) x_{n}(t)+c_{n, n-1}(t) x_{n}(t)\right)-h_{n}(t) .
\end{aligned}
$$

As we know, in population dynamics, many evolutionary processes experience short-time rapid chance after undergoing relatively long sooth variation. Examples include stocking of species and annual immigration. Incorporating these phenomena give us impulsive differential equations. For the theory of impulsive differential equations, we refer the reader to $[11,12]$. In addition, there are few results on the existence of multiple periodic solutions for the delay food-chain system with impulsive effects in literatures. This motivates us to modify system (1.1) to the form

$$
\begin{aligned}
\dot{x}_{1}(t)= & x_{1}(t)\left(a_{1}(t)-b_{1}(t) x_{1}\left(t-\tau_{11}(t)\right)-c_{12}(t) x_{2}\left(t-\tau_{12}(t)\right)\right)-h_{1}(t), \quad t \neq t_{k}, \\
\vdots & \\
\dot{x}_{i}(t)= & x_{i}(t)\left(-d_{i}(t)-b_{i}(t) x_{i}\left(t-\tau_{i i}(t)\right)+c_{i, i-1}(t) x_{i-1}\left(t-\tau_{i, i-1}(t)\right)-c_{i, i+1}(t) x_{i+1}\left(t-\tau_{i, i+1}(t)\right)\right) \\
& \quad-h_{i}(t), \quad 2 \leq i \leq n-1, t \neq t_{k}, \\
& \vdots \\
\dot{x}_{n}(t)= & x_{n}(t)\left(-d_{n}(t)-b_{n}(t) x_{n}\left(t-\tau_{n n}(t)\right)+c_{n, n-1}(t) x_{n}\left(t-\tau_{n, n-1}(t)\right)\right)-h_{n}(t), \quad t \neq t_{k}, \\
\Delta x_{i}\left(t_{k}\right)= & x_{i}\left(t_{k}^{+}\right)-x_{i}\left(t_{k}^{-}\right)=g_{i k} x_{i}\left(t_{k}\right), \quad 1 \leq i \leq n,
\end{aligned}
$$

where $g_{i k} \in(-1,+\infty)(i=1,2, \ldots, n ; k \in \mathbb{N}=\{1,2, \ldots, n\}) .\left\{t_{k}\right\}_{k \in \mathbb{N}}$ is a strictly increasing sequence with $t_{1}>0$ and $\lim _{t \rightarrow \infty} t_{k}=\infty \cdot x_{i}(t)(i=1,2, \ldots, n)$ is the $i$ th species population density. $a_{1}(t)>0$ is the growth rate of the only producer. $b_{i}(t)>0(i=1,2, \ldots, n)$ and $h_{i}(t)>0(i=1,2, \ldots, n)$ stand for the $i$ th species intraspecific competition rate and harvesting rate, respectively. $d_{i}(t)>0(i=2,3, \ldots, n)$ is the $i$ th species death rate. $c_{i, i+1}(t)>0(i=$ $1,2, \ldots, n-1)$ represents the $(i+1)$ th species predation rate on the $i$ th species. $c_{i, i-1}(t)>0(i=$ $2,3, \ldots, n)$ stands for the transformation rate from the $(i-1)$ th species to the $i$ th species. $\tau_{i, i+1}(t) \geq 0(i=1,2, \ldots, n-1)$ stands for the time-lag in the process of the $(i+1)$ th species predation rate on the $i$ th species. $\tau_{i, i-1}(t) \geq 0(i=2,3, \ldots, n)$ stands for the time-lag in the process of transformation from the $(i-1)$ th species to the $i$ th species. $\tau_{i i}(t) \geq 0(i=1,2, \ldots, n)$ represents the time-lag in the process of intraspecific competition. In addition, the effects of a periodically varying environment are important for evolutionary theory as the selective forces on systems in a fluctuating environment differ from those in a stable environment. Therefore, the assumptions of periodicity of the parameters are a way of incorporating the periodicity of the environment (e.g., seasonal effects of weather, food supplies, mating habits, etc.), which leads us to assume that $a_{i}(t), b_{i}(t), c_{i j}(t), \tau_{i j}(t)$, and $h_{i}(t)(i, j=1,2, \ldots, n)$ are all 
continuous $\omega$-periodic functions. For impulsive effects, we further assume that there exists a $q \in \mathbb{N}$ such that $g_{i(k+q)}=g_{i k}(i=1,2, \ldots, n)$ and $t_{k+q}=t_{k}+\omega$.

However, dynamics in each equally spaced time interval may vary continuously. So, it may be more realistic to assume that the population dynamics involves the hybrid discrete-continuous processes. For example, Gamarra and Sole pointed out that such hybrid processes appear in the population dynamics of certain species that feature nonoverlapping generations: the change in population from one generation to the next is discrete and so is modeled by a difference equation, while within-generation dynamics vary continuously (due to mortality rates, resource consumption, predation, interaction, etc.) and thus are described by a differential equation [13]. The theory of calculus on time scales (see [14, 15] and references cited therein) was initiated by Hilger in his Ph.D. thesis in 1988 [16] in order to unify continuous and discrete analysis, and it has become an effective approach to the study of mathematical models involving the hybrid discrete-continuous processes. This motivates us to consider Lotka-Volterra food-chain system with delays and impulses on time scales $\mathbb{T}$ of the following form:

$$
\begin{aligned}
& u_{1}^{\Delta}(t)=a_{1}(t)-b_{1}(t) e^{u_{1}\left(t-\tau_{11}(t)\right)}-c_{12}(t) e^{u_{2}\left(t-\tau_{12}(t)\right)}-h_{1}(t) e^{-u_{1}(t)}, \quad t \in \mathbb{T}, t \neq t_{k}, \\
& \vdots \\
& u_{i}^{\Delta}(t)=-d_{i}(t)-b_{i}(t) e^{u_{i}\left(t-\tau_{i i}(t)\right)}+c_{i, i-1}(t) e^{u_{i-1}\left(t-\tau_{i, i-1}(t)\right)}-c_{i, i+1}(t) e^{u_{i+1}\left(t-\tau_{i, i+1}(t)\right)} \\
& -h_{i}(t) e^{-u_{i}(t)}, \quad 2 \leq i \leq n-1, t \in \mathbb{T}, t \neq t_{k}, \\
& u_{n}^{\Delta}(t)=-d_{n}(t)-b_{n}(t) e^{u_{n}\left(t-\tau_{n n}(t)\right)}+c_{n, n-1}(t) e^{u_{n}\left(t-\tau_{n, n-1}(t)\right)}-h_{n}(t) e^{-u_{n}(t)}, \quad t \in \mathbb{T}, t \neq t_{k} ， \\
& \Delta u_{i}\left(t_{k}\right)=u_{i}\left(t_{k}^{+}\right)-u_{i}\left(t_{k}^{-}\right)=\ln \left(1+g_{i k}\right), \quad 1 \leq i \leq n,
\end{aligned}
$$

where $\mathbb{T}$ is a $\omega$-periodic time scale. $g_{i k} \in(-1,+\infty)(i=1,2, \ldots, n ; k \in \mathbb{N}=\{1,2, \ldots, n\})$ is a constant. $\left\{t_{k}\right\}_{k \in \mathbb{N}}$ is a strictly increasing sequence with $t_{1}>0$ and $\lim _{k \rightarrow \infty} t_{k}=\infty \cdot a_{1}(t)>0$, $b_{i}(t)>0, h_{i}(t)>0, d_{i}(t)>0, c_{i, i+1}(t)>0, c_{i, i-1}(t)>0, \tau_{i, i+1}(t), \tau_{i, i-1}(t), \tau_{i i}(t) \geq 0$ are rdcontinuous $\omega$-periodic functions. For impulsive effects, we further assume that there exists a integer $q \in \mathbb{N}$ such that $g_{i(k+q)}=g_{i k}(i=1,2, \ldots, n)$ and $t_{k+q}=t_{k}+\omega$. We denote by $\kappa=$ $\min \{[0,+\infty) \cap \mathbb{T}\}, I_{\omega}=[\kappa, \kappa+\omega] \cap \mathbb{T}$. Without loss of generality, we also assume that $I_{\omega} \cap\left\{t_{k}\right.$ : $k \in \mathbb{N}\}=\left\{t_{1}, t_{2}, \ldots, t_{q}\right\}$.

Remark 1.1. In (1.3), let $x_{i}(t)=e^{u_{i}(t)}, i=1,2, \ldots, n$. If $\mathbb{T}=\mathbb{R}$ (the set of all real numbers), then (1.3) reduces to (1.2).

To the best our knowledge, few papers have been published on the existence of multiple periodic solutions for this model. Our main purpose of this paper is by using Mawhin's continuation theorem of coincidence degree theory [17], to establish the existence of at least $2^{n}$ periodic solutions for system (1.3). For the work concerning the multiple existence of periodic solutions of periodic population models which was done using coincidence degree theory, we refer to [18-25]. 
The organization of the rest of this paper is as follows. In the Section 2, we will introduce some basic notations and lemmas which are used in what follows. In Section 3, by employing the continuation theorem of coincidence degree theory and the skills of inequalities, we establish the existence of at least $2^{n}$ periodic solutions of system (1.3). In the final Section, one example is given to illustrate the effectiveness of our results.

\section{Preliminaries on Time Scales}

In this section, we briefly recall some basic definitions and lemmas on time scales which are used in what follows. For more details, one can see [14-16].

Let $\mathbb{T}$ be a nonempty closed subset (time scale) of $\mathbb{R}$. The forward and backward jump operators $\sigma, \rho: \mathbb{T} \rightarrow \mathbb{T}$ and the graininess $\mu: \mathbb{T} \rightarrow \mathbb{R}^{+}$are defined, respectively, by

$$
\sigma(t)=\inf \{s \in \mathbb{T}: s>t\}, \quad \rho(t)=\sup \{s \in \mathbb{T}: s<t\}, \quad \mu(t)=\sigma(t)-t .
$$

A point $t \in \mathbb{T}$ is called left-dense if $t>\inf \mathbb{T}$ and $\rho(t)=t$, left-scattered if $\rho(t)<t$, right-dense if $t<\sup \mathbb{T}$ and $\sigma(t)=t$, and right-scattered if $\sigma(t)>t$. If $\mathbb{T}$ has a left-scattered maximum $m$, then $\mathbb{T}^{k}=\mathbb{T} \backslash\{m\}$; otherwise $\mathbb{T}^{k}=\mathbb{T}$. If $\mathbb{T}$ has a right-scattered minimum $m$, then $\mathbb{T}_{k}=\mathbb{T} \backslash\{m\}$; otherwise $\mathbb{T}^{k}=\mathbb{T}$.

Let $\omega>0$. Throughout this paper, the time scale $\mathbb{T}$ is assumed to be $\omega$-periodic, that is, $t \in \mathbb{T}$ implies $t+\omega \in \mathbb{T}$. In particular, the time scale $\mathbb{T}$ under consideration is unbounded above and below.

Definition 2.1. A function $f: \mathbb{T} \rightarrow \mathbb{R}$ is called regulated provided its right-side limits exist (finite) at all right-side points in $\mathbb{T}$ and its left-side limits exist (finite) at all left-side points in $\mathbb{T}$.

Definition 2.2. A function $f: \mathbb{T} \rightarrow \mathbb{R}$ is called rd-continuous provided it is continuous at right-dense point in $\mathbb{T}$ and its left-side limits exist (finite) at left-dense points in $\mathbb{T}$. The set of rd-continuous functions $f: \mathbb{T} \rightarrow \mathbb{R}$ will be denoted by $C_{\mathrm{rd}}=C_{\mathrm{rd}}(\mathbb{T})=C_{\mathrm{rd}}(\mathbb{T}, \mathbb{R})$.

Definition 2.3. Assume $f: \mathbb{T} \rightarrow \mathbb{R}$ and $t \in \mathbb{T}^{k}$. Then we define $f^{\Delta}(t)$ to be the number (if it exists) with the property that given any $\varepsilon>0$ there exists a neighborhood $U$ of $t$ (i.e., $U=(t-\delta, t+\delta) \cap \mathbb{T}$ for some $\delta>0)$ such that

$$
\left|[f(\sigma(t))-f(s)]-f^{\Delta}(t)[\sigma(t)-s]\right|<\varepsilon|\sigma(t)-s|
$$

for all $s \in U$. we call $f^{\Delta}(t)$ the delta (or Hilger) derivative of $f$ at $t$. The set of functions $f: \mathbb{T} \rightarrow \mathbb{R}$ that are differentiable and whose derivative is rd-continuous is denoted by $C_{\mathrm{rd}}^{1}=$ $C_{\mathrm{rd}}^{1}(\mathbb{T})=C_{\mathrm{rd}}^{1}(\mathbb{T}, \mathbb{R})$.

If $f$ is continuous, then $f$ is rd-continuous. If $f$ is rd-continuous, the $f$ is regulated. If $f$ is delta differentiable at $t$, then $f$ is continuous at $t$. 
Lemma 2.4. Let $f$ be regulated, then there exists a function $F$ which is delta differentiable with region of differentiation $D$ such that

$$
F^{\Delta}(t)=f(t) \quad \forall t \in D
$$

Definition 2.5. Assume $f: \mathbb{T} \rightarrow \mathbb{R}$ is a regulated function. Any function $F$ as in Lemma 2.4 is called a $\Delta$-antiderivative of $f$. We define the indefinite integral of a regulated function $f$ by

$$
\int f(t) \Delta t=F(t)+C
$$

where $C$ is an arbitrary constant and $F$ is a $\Delta$-antiderivative of $f$. We define the Cauchy integral by

$$
\int_{a}^{b} f(s) \Delta s=F(b)-F(a) \quad \forall a, b \in \mathbb{T}
$$

A function $F: \mathbb{T} \rightarrow \mathbb{R}$ is called an antiderivative of $f: \mathbb{T} \rightarrow \mathbb{R}$ provided

$$
F^{\Delta}(t)=f(t) \quad \forall t \in \mathbb{T}^{k}
$$

Lemma 2.6. If $a, b \in \mathbb{T}, \alpha, \beta \in \mathbb{R}$ and $f, g \in C(\mathbb{T}, \mathbb{R})$, then

(i) $\int_{a}^{b}[\alpha f(t)+\beta g(t)] \Delta t=\alpha \int_{a}^{b} f(t) \Delta t+\beta \int_{a}^{b} g(t) \Delta t$;

(ii) if $f(t) \geq 0$ for all $a \leq t<b$, then $\int_{a}^{b} f(t) \Delta t \geq 0$;

(iii) if $|f(t)| \leq g(t)$ on $[a, b):=\{t \in \mathbb{T}: a \leq t<b\}$, then $\left|\int_{a}^{b} f(t) \Delta t\right| \leq \int_{a}^{b} g(t) \Delta t$. Let

For convenience, we now introduce some notation to be used throughout this paper.

$$
\begin{gathered}
\mathcal{\kappa}=\min \{[0,+\infty) \cap \mathbb{T}\}, \quad I_{\omega}=[\kappa, \kappa+\omega] \cap \mathbb{T}, \quad g^{M}=\sup _{t \in I_{\omega}} g(t), \\
g^{l}=\inf _{t \in I_{\omega}} g(t), \quad \bar{g}=\frac{1}{\omega} \int_{I_{\omega}} g(s) \Delta s=\frac{1}{\omega} \int_{\kappa}^{\kappa+\omega} g(s) \Delta s,
\end{gathered}
$$

where $g \in C_{\mathrm{rd}}(\mathbb{T})$ is an $\omega$-periodic real function, that is, $g(t+\omega)=g(t)$ for all $t \in \mathbb{T}$.

Lemma 2.7 (see [26]). Assume that $\left\{f_{n}\right\}_{n \in N}$ is a function on J such that

(i) $\left\{f_{n}\right\}_{n \in N}$ is uniformly bounded on $J$,

(ii) $\left\{f_{n}^{\Delta}\right\}_{n \in N}$ is uniformly bounded on $J$.

Then there is a subsequence of $\left\{f_{n}\right\}_{n \in N}$ which converges uniformly on J. 


\section{Existence of at Least $2^{n}$ Periodic Solutions}

In this section, by using Mawhin's continuation theorem and the skills of inequalities, we will show the existence of periodic solutions of (1.3). To do so, we need to make some preparations.

Let $X$ and $Z$ be real normed vector spaces. Let $L:$ Dom $L \subset X \rightarrow Z$ be a linear mapping and $N: X \times[0,1] \rightarrow Z$ be a continuous mapping. The mapping $L$ will be called a Fredholm mapping of index zero if $\operatorname{dim} \operatorname{Ker} L=$ codim $\operatorname{Im} L<\infty$ and $\operatorname{Im} L$ is closed in $Z$. If $L$ is a Fredholm mapping of index zero, then there exists continuous projectors $P: X \rightarrow X$ and $Q: Z \rightarrow Z$ such that $\operatorname{Im} P=\operatorname{Ker} L$ and $\operatorname{Ker} Q=\operatorname{Im} L=\operatorname{Im}(I-Q)$, and $X=\operatorname{Ker} L \oplus \operatorname{Ker} P$, $Z=\operatorname{Im} L \oplus \operatorname{Im} Q$. It follows that $\left.L\right|_{\operatorname{Dom} L \cap K e r} P:(I-P) X \rightarrow \operatorname{Im} L$ is invertible and its inverse is denoted by $K_{P}$. If $\Omega$ is a bounded open subset of $X$, the mapping $N$ is called $L$-compact on $\bar{\Omega} \times[0,1]$, if $Q N(\bar{\Omega} \times[0,1])$ is bounded and $K_{P}(I-Q) N: \bar{\Omega} \times[0,1] \rightarrow X$ is compact. Because $\operatorname{Im} Q$ is isomorphic to $\operatorname{Ker} L$, there exists an isomorphism $J: \operatorname{Im} Q \rightarrow \operatorname{Ker} L$.

The Mawhin's continuous theorem [17, page 40] is given as follows.

Lemma 3.1 (see [17]). Let $L$ be a Fredholm mapping of index zero and let $N$ be L-compact on $\bar{\Omega} \times[0,1]$. Assume

(a) for each $\lambda \in(0,1)$, every solution $x$ of $L x=\lambda N(x, \lambda)$ is such that $x \notin \partial \Omega \cap \operatorname{Dom} L$;

(b) $Q N(x, 0) x \neq 0$ for each $x \in \partial \Omega \cap \operatorname{Ker} L$;

(c) $\operatorname{deg}(J Q N(x, 0), \Omega \cap \operatorname{Ker} L, 0) \neq 0$.

Then $L x=N(x, 1)$ has at least one solution in $\bar{\Omega} \cap \operatorname{Dom} L$.

For simplicity, we need to introduce some notations as follows:

$$
\begin{gathered}
g_{i}=\frac{1}{2} \sum_{k=1}^{q} \ln \left(1+g_{i k}\right) \quad(1 \leq i \leq n), \quad G_{i}=\frac{1}{2} \sum_{k=1}^{q}\left[\ln \left(1+g_{i k}\right)+\left|\ln \left(1+g_{i k}\right)\right|\right] \quad(1 \leq i \leq n), \\
l_{1}^{ \pm}=\frac{\left(\bar{a}_{1}+\left(2 g_{1} / \omega\right)\right) e^{\bar{a}_{1} \omega+G_{1}} \pm \sqrt{\left(\left(\bar{a}_{1}+\left(2 g_{1} / \omega\right)\right) e^{\bar{a}_{1} \omega+G_{1}}\right)^{2}-4 \bar{b}_{1} \bar{h}_{1} e^{\bar{a}_{1} \omega+G_{1}}}}{2 \bar{b}_{1}}, \\
A_{1}^{ \pm}=\frac{\left(\bar{a}_{1}+\left(2 g_{1} / \omega\right)-\bar{c}_{12} l_{2}^{+}\right) \pm \sqrt{\left(\bar{a}_{1}+\left(2 g_{1} / \omega\right)-\bar{c}_{12} l_{2}^{+}\right)^{2}-4 \bar{b}_{1} \bar{h}_{1} e^{\bar{a}_{1} \omega+G_{1}}}}{2 \bar{b}_{1}}, \\
B_{1}^{ \pm}=\frac{\left(\bar{a}_{1}+\left(2 g_{1} / \omega\right)-\bar{c}_{12} l_{2}^{+}\right) e^{-\left(\bar{a}_{1} \omega+G_{1}\right)} \pm \sqrt{\left(\left(\bar{a}_{1}+\left(2 g_{1} / \omega\right)-\bar{c}_{12} l_{2}^{+}\right) e^{-\left(\bar{a}_{1} \omega+G_{1}\right)}\right)^{2}-4 \bar{b}_{1} \bar{h}_{1} e^{-\left(\bar{a}_{1} \omega+G_{1}\right)}}}{2 \bar{b}_{1}}, \\
2 l_{i}^{ \pm}=\frac{\left(\bar{c}_{i, i-1}+\left(2 g_{i} / \omega\right)\right) e^{\bar{c}_{i, i-1} \omega+G_{i}} \pm \sqrt{\left(\left(\bar{c}_{i, i-1}+\left(2 g_{i} / \omega\right)\right) e^{\bar{c}_{i, i-1} \omega+G_{i}}\right)^{2}-4 \bar{b}_{i} \bar{h}_{i} e^{\bar{c}_{i, i-1} \omega+G_{i}}}}{(2 \leq n),}
\end{gathered}
$$




$$
\begin{gathered}
A_{i}^{ \pm}=\frac{\left(\left(2 g_{i} / \omega\right)-\bar{d}_{i}-\bar{c}_{i, i+1} l_{i+1}^{+}\right) \pm \sqrt{\left(\left(2 g_{i} / \omega\right)-\bar{d}_{i}-\bar{c}_{i, i+1} l_{i+1}^{+}\right)^{2}-4 \bar{b}_{i} \bar{h}_{i} e^{\bar{c}_{i, i-1} \omega+G_{i}}}}{2 \bar{b}_{i}} \quad(2 \leq i \leq n-1), \\
B_{i}^{ \pm}=\frac{1}{2 \bar{b}_{i}}\left[ \pm \sqrt{\left(\left(\frac{2 g_{i}}{\omega}-\bar{d}_{i}-\bar{c}_{i, i+1} l_{i+1}^{+}\right) e^{-\left(\bar{c}_{i,-1} \omega+G_{i}\right)}\right)^{2}-4 \bar{b}_{i} \bar{h}_{i} e^{-\left(\bar{c}_{i, i-1} \omega+G_{i}\right)}}\right. \\
B_{n}^{ \pm}=\frac{\left.+\left(\frac{2 g_{i}}{\omega}-\bar{d}_{i}-\bar{c}_{i, i+1} l_{i+1}^{+}\right) e^{-\left(\bar{c}_{i, i-1} \omega+G_{i}\right)}\right] \quad(2 \leq i \leq n-1),}{\left.\left.2 g_{n} / \omega\right)-\bar{d}_{n}\right) e^{-\left(\bar{c}_{n, n-1} \omega+G_{n}\right)} \pm \sqrt{\left(\left(\left(2 g_{n} / \omega\right)-\bar{d}_{n}\right) e^{\left.-\left(\bar{c}_{n, n-1} \omega+G_{n}\right)\right)^{2}-4 \bar{b}_{n}} \bar{h}_{n} e^{-\left(\bar{c}_{n, n-1} \omega+G_{n}\right)}\right.}} \\
A_{n}^{ \pm}=\frac{\left(\left(2 g_{n} / \omega\right)-\bar{d}_{n}\right) \pm \sqrt{\left(\left(2 g_{n} / \omega\right)-\bar{d}_{n}\right)^{2}-4 \bar{b}_{n} \bar{h}_{n} e^{\bar{c}_{n, n-1} \omega+G_{n}}}}{2 \bar{b}_{n}}, \\
H_{1}=l_{1}^{-} e^{-\left(\bar{a}_{1} \omega+G_{1}\right)}, \quad H_{i}=l_{i}^{-} e^{-\left(\bar{c}_{i, i-1} \omega+G_{i}\right)} \quad(2 \leq i \leq n) .
\end{gathered}
$$

Throughout this paper, we need the following assumptions:

$$
\begin{aligned}
& \left(H_{1}\right)\left(\bar{a}_{1}+\left(2 g_{1} / \omega\right)-\bar{c}_{12} l_{2}^{+}\right) e^{-(1 / 2)\left(\bar{a}_{1} \omega+G_{1}\right)}>2 \sqrt{\bar{b}_{1} \bar{h}_{1}} \\
& \left(H_{2}\right)\left(\left(2 g_{i} / \omega\right)-\bar{d}_{i}-\bar{c}_{i, i+1} l_{i+1}^{+}\right) e^{-(1 / 2)\left(\bar{c}_{i, i-1} \omega+G_{i}\right)}>2 \sqrt{\bar{b}_{i} \bar{h}_{i}}(2 \leq i \leq n-1), \\
& \left(H_{3}\right)\left(\left(2 g_{n} / \omega\right)-\bar{d}_{n}\right) e^{-(1 / 2)\left(\bar{c}_{n, n-1} \omega+G_{n}\right)}>2 \sqrt{\bar{b}_{n} \bar{h}_{n}}
\end{aligned}
$$

The following results will play an important role in the proof of our main result.

Lemma 3.2. Let $\mathbb{T}$ be a w-periodic time scale. $\left\{t_{k}\right\}_{1}^{\infty} \subset \mathbb{T}$ is a strictly increasing sequence with $t_{1}>0$ and $\lim _{k \rightarrow \infty} t_{k}=\infty . \kappa=\min \{[0, \infty) \cap \mathbb{T}\}, I_{\omega}=[\kappa, \kappa+\omega] \cap \mathbb{T}, I_{\omega} \cap\left\{t_{k}\right\}_{k=1}^{\infty}=\left\{t_{1}, t_{2}, \ldots, t_{q}\right\}$. Suppose $\psi: \mathbb{T} \rightarrow \mathbb{R}$ be a w-periodic function which is rd-continuous for $t \neq t_{k}$, exists the right limit $\psi\left(t_{k}^{+}\right)$and left limit $\psi\left(t_{k}^{-}\right)=\psi\left(t_{k}\right)$ in the sense of time scales. Then

$$
0 \leq \sup _{s \in I_{\omega}} \psi(s)-\inf _{s \in I_{\omega}} \psi(s) \leq \frac{1}{2}\left[\int_{\kappa}^{\mathcal{\kappa}+\omega}\left|\psi^{\Delta}(s)\right| \Delta s+\sum_{k=1}^{q}\left|\Delta \psi\left(t_{k}\right)\right|\right] .
$$


Proof. First, take $t_{*} \in I_{\omega}$ such that $\psi\left(t_{*}\right)=\inf _{s \in I_{\omega}} \psi(s)$. Next, take $t^{*} \in\left[t_{*}, t_{*}+\omega\right] \cap \mathbb{T}$ such that $\psi\left(t^{*}\right)=\sup _{s \in I_{\omega}} \psi(s)$. Then we have

$$
\begin{aligned}
\psi\left(t^{*}\right) & =\psi\left(t_{*}\right)+\left(\psi\left(t^{*}\right)-\psi\left(t_{*}\right)\right) \\
& =\psi\left(t_{*}\right)+\left(\psi\left(t^{*}\right)-\sum_{t_{*} \leq t_{k}<t^{*}} \Delta \psi\left(t_{k}\right)-\psi\left(t_{*}\right)\right)+\sum_{t_{*} \leq t_{k}<t^{*}} \Delta \psi\left(t_{k}\right) \\
& =\psi\left(t_{*}\right)+\int_{t_{*}}^{t^{*}} \psi(s) \Delta s+\sum_{t_{*} \leq t_{k}<t^{*}} \Delta \psi\left(t_{k}\right) \\
& \leq \psi\left(t_{*}\right)+\int_{t_{*}}^{t^{*}}|\psi(s)| \Delta s+\sum_{t_{*} \leq t_{k}<t^{*}}\left|\Delta \psi\left(t_{k}\right)\right| .
\end{aligned}
$$

Noticing that $\psi\left(t^{*}\right)=\psi\left(t^{*}-\omega\right)=\psi\left(t_{*}\right)+\left(\psi\left(t^{*}\right)-\psi\left(t_{*}-\omega\right)\right)$, similar to the above, we can get

$$
\psi\left(t^{*}\right) \leq \psi\left(t_{*}\right)+\int_{t^{*}-\omega}^{t_{*}}|\psi(s)| \Delta s+\sum_{t^{*}-\omega \leq t_{k}<t_{*}}\left|\Delta \psi\left(t_{k}\right)\right| .
$$

From (3.3) and (3.4), we get

$$
\begin{aligned}
2 \psi\left(t^{*}\right) & \leq 2 \psi\left(t_{*}\right)+\int_{t_{*}}^{t^{*}}|\psi(s)| \Delta s+\int_{t^{*}-\omega}^{t_{*}}|\psi(s)| \Delta s+\sum_{t_{*} \leq t_{k}<t^{*}}\left|\Delta \psi\left(t_{k}\right)\right|+\sum_{t^{*}-\omega \leq t_{k}<t_{*}}\left|\Delta \psi\left(t_{k}\right)\right| \\
& =2 \psi\left(t_{*}\right)+\int_{0}^{\omega}|\psi(s)| \Delta s+\sum_{0 \leq t_{k}<\omega}\left|\Delta \psi\left(t_{k}\right)\right|
\end{aligned}
$$

that is

$$
\psi\left(t^{*}\right) \leq \psi\left(t_{*}\right)+\frac{1}{2}\left[\int_{\kappa}^{\kappa+\omega}|\psi(s)| \Delta s+\sum_{k=1}^{q}\left|\Delta \psi\left(t_{k}\right)\right|\right]
$$

which implies that (3.2) holds. This completes the proof.

Lemma 3.3. Let $x>0, y>0, z>0$ and $x>2 \sqrt{y z}$, for the functions $f(x, y, z)=(x+$ $\left.\sqrt{x^{2}-4 y z}\right) / 2 z$ and $g(x, y, z)=\left(x-\sqrt{x^{2}-4 y z}\right) / 2 z$, the following assertions hold.

(1) $f(x, y, z)$ and $g(x, y, z)$ are monotonically increasing and monotonically decreasing on the variable $x \in(0, \infty)$, respectively.

(2) $f(x, y, z)$ and $g(x, y, z)$ are monotonically decreasing and monotonically increasing on the variable $y \in(0, \infty)$, respectively.

(3) $f(x, y, z)$ and $g(x, y, z)$ are monotonically decreasing and monotonically increasing on the variable $z \in(0, \infty)$, respectively. 
Proof. In fact, for all $x>0, y>0, z>0$, we have

$$
\begin{gathered}
\frac{\partial f}{\partial x}=\frac{x+\sqrt{x^{2}-4 y z}}{2 z \sqrt{x^{2}-4 y z}}>0, \quad \frac{\partial g}{\partial x}=\frac{\sqrt{x^{2}-4 y z}-x}{2 z \sqrt{x^{2}-4 y z}}<0, \quad \frac{\partial f}{\partial y}=\frac{-1}{\sqrt{x^{2}-4 y z}}<0, \\
\frac{\partial g}{\partial y}=\frac{1}{\sqrt{x^{2}-4 y z}}>0, \quad \frac{\partial f}{\partial z}=\frac{-\left(x+\sqrt{x^{2}-4 y z}\right)^{2}}{4 z^{2} \sqrt{x^{2}-4 y z}}<0, \quad \frac{\partial g}{\partial z}=\frac{\left(x-\sqrt{x^{2}-4 y z}\right)^{2}}{4 z^{2} \sqrt{x^{2}-4 y z}}>0 .
\end{gathered}
$$

By the relationship of the derivative and the monotonicity, the above assertions obviously hold. The proof of Lemma 3.3 is complete.

Lemma 3.4. Assume that $\left(H_{1}\right),\left(H_{2}\right)$, and $\left(H_{3}\right)$ hold, then we have the following inequalities:

(1) $\ln l_{i}^{+}>\ln A_{i}^{+}>\ln A_{i}^{-}>\ln l_{i}^{-}>\ln H_{i}(1 \leq i \leq n)$;

(2) $\ln l_{i}^{+}>\ln B_{i}^{+}>\ln B_{i}^{-}>\ln l_{i}^{-}>\ln H_{i}(1 \leq i \leq n)$;

(3) $\min \left\{\ln A_{i}^{+}, \ln B_{i}^{+}\right\}>\max \left\{\ln A_{i}^{-}, \ln B_{i}^{-}\right\}(1 \leq i \leq n)$.

Proof. (1) Since

$$
\begin{aligned}
\left(\bar{a}_{1}+\frac{2 g_{1}}{\omega}\right) e^{\bar{a}_{1} \omega+G_{1}}>\bar{a}_{1}+ & \frac{2 g_{1}}{\omega}-\bar{c}_{12} l_{2}^{+}>\left(\bar{a}_{1}+\frac{2 g_{1}}{\omega}-\bar{c}_{12} l_{2}^{+}\right) e^{-(1 / 2)\left(\bar{a}_{1} \omega+G_{1}\right)}>2 \sqrt{\bar{b}_{1} \bar{h}_{1}}>0, \\
\left(\bar{c}_{i, i-1}+\frac{2 g_{i}}{\omega}\right) e^{\bar{c}_{i, i-1} \omega+G_{i}} & >\frac{2 g_{i}}{\omega}-\bar{d}_{i}-\bar{c}_{i, i+1} l_{i+1}^{+}>\left(\frac{2 g_{i}}{\omega}-\bar{d}_{i}-\bar{c}_{i, i+1} l_{i+1}^{+}\right) e^{-(1 / 2)\left(\bar{c}_{i, i-1} \omega+G_{i}\right)} \\
> & 2 \sqrt{\bar{b}_{i} \bar{h}_{i}}>0, \\
\left(\bar{c}_{n, n-1}+\frac{2 g_{n}}{\omega}\right) e^{\bar{c}_{n, n-1} \omega+G_{n}} & >\frac{2 g_{n}}{\omega}-\bar{d}_{n}>\left(\frac{2 g_{n}}{\omega}-\bar{d}_{n}\right) e^{-(1 / 2)\left(\bar{c}_{n, n-1} \omega+G_{n}\right)}>2 \sqrt{\bar{b}_{n} \bar{h}_{n}}>0, \\
0 & <\bar{b}_{i}, \quad 0<\bar{h}_{i}, \quad i=1,2, \ldots, n .
\end{aligned}
$$


Applying Lemma 3.3, we have

$$
\begin{aligned}
& 0<H_{1}=l_{1}^{-} e^{-\left(\bar{a}_{1} \omega+G_{1}\right)}<l_{1}^{-}=g\left(\left(\bar{a}_{1}+\frac{2 g_{1}}{\omega}\right) e^{\bar{a}_{1} \omega+G_{1}}, \bar{b}_{1}, \bar{h}_{1} e^{\bar{a}_{1} \omega+G_{1}}\right) \\
& <g\left(\bar{a}_{1}+\frac{2 g_{1}}{\omega}-\bar{c}_{12} l_{2}^{+}, \bar{b}_{1}, \bar{h}_{1} e^{a_{1} \omega+G_{1}}\right)=A_{1}^{-}<A_{1}^{+}=f\left(\bar{a}_{1}+\frac{2 g_{1}}{\omega}-\bar{c}_{12} l_{2}^{+}, \bar{b}_{1}, \bar{h}_{1} e^{\bar{a}_{1} \omega+G_{1}}\right) \\
& <f\left(\left(\bar{a}_{1}+\frac{2 g_{1}}{\omega}\right) e^{\bar{a}_{1} \omega+G_{1}}, \bar{b}_{1}, \bar{h}_{1} e^{\bar{a}_{1} \omega+G_{1}}\right)=l_{1}^{+}, \\
& 0<H_{i}=l_{i}^{-} e^{-\left(\bar{c}_{i, i-1} \omega+G_{i}\right)}<l_{i}^{-}=g\left(\left(\bar{c}_{i, i-1}+\frac{2 g_{i}}{\omega}\right) e^{\bar{c}_{i, i-1} \omega+G_{i}}, \bar{b}_{i}, \bar{h}_{i} e^{\bar{c}_{i, i-1} \omega+G_{i}}\right) \\
& <g\left(\frac{2 g_{i}}{\omega}-\bar{d}_{i}-\bar{c}_{i, i+1} l_{i+1}^{+}, \bar{b}_{i}, \bar{h}_{i} e^{\bar{c}_{i, i-1} \omega+G_{i}}\right)=A_{i}^{-} \\
& <A_{i}^{+}=f\left(\frac{2 g_{i}}{\omega}-\bar{d}_{i}-\bar{c}_{i, i+1} l_{i+1}^{+}, \bar{b}_{i}, \bar{h}_{i} e^{\bar{c}_{i, i-1} \omega+G_{i}}\right) \\
& <f\left(\left(\bar{c}_{i, i-1}+\frac{2 g_{i}}{\omega}\right) e^{\bar{c}_{i, i-1} \omega+G_{i}}, \bar{b}_{i}, \bar{h}_{i} e^{\bar{c}_{i, i-1} \omega+G_{i}}\right)=l_{i}^{+}, \quad i=2,3, \ldots, n-1, \\
& 0<H_{n}=l_{n}^{-} e^{-\left(\bar{c}_{n, n-1} \omega+G_{n}\right)}<l_{n}^{-}=g\left(\left(\bar{c}_{n, n-1}+\frac{2 g_{n}}{\omega}\right) e^{\bar{c}_{n, n-1} \omega+G_{n}}, \bar{b}_{n}, \bar{h}_{n} e^{\bar{c}_{n, n-1} \omega+G_{n}}\right) \\
& <g\left(\frac{2 g_{n}}{\omega}-\bar{d}_{n}, \bar{b}_{n}, \bar{h}_{n} e^{\bar{c}_{n, n-1} \omega+G_{n}}\right)=A_{n}^{-}<A_{n}^{+}=f\left(\frac{2 g_{n}}{\omega}-\bar{d}_{n}, \bar{b}_{n}, \bar{h}_{n} e^{\bar{c}_{n, n-1} \omega+G_{n}}\right) \\
& <f\left(\left(\bar{c}_{n, n-1}+\frac{2 g_{n}}{\omega}\right) e^{\bar{c}_{n, n-1} \omega+G_{n}}, \bar{b}_{n}, \bar{h}_{n} e^{\bar{c}_{n, n-1} \omega+G_{n}}\right)=l_{n}^{+} .
\end{aligned}
$$

Thus, we have $\ln H_{i}<\ln l_{i}^{-}<\ln A_{i}^{-}<\ln A_{i}^{+}<\ln l_{i}^{+}, i=1,2, \ldots, n$. The proof of (1) of Lemma 3.4 is complete. For (2) of Lemma 3.4, we similarly have

$$
\begin{aligned}
0 & <H_{1}=l_{1}^{-} e^{-\left(\bar{a}_{1} \omega+G_{1}\right)}<l_{1}^{-}=g\left(\left(\bar{a}_{1}+\frac{2 g_{1}}{\omega}\right) e^{\bar{a}_{1} \omega+G_{1}}, \bar{b}_{1} e^{\bar{a}_{1} \omega+G_{1}}, \bar{h}_{1}\right) \\
& <g\left(\bar{a}_{1}+\frac{2 g_{1}}{\omega}-\bar{c}_{12} l_{2}^{+}, \bar{b}_{1} e^{a_{1} \omega+G_{1}}, \bar{h}_{1}\right)=B_{1}^{-}<B_{1}^{+}=f\left(\bar{a}_{1}+\frac{2 g_{1}}{\omega}-\bar{c}_{12} l_{2}^{+}, \bar{b}_{1} e^{\bar{a}_{1} \omega+G_{1}}, \bar{h}_{1}\right) \\
& <f\left(\left(\bar{a}_{1}+\frac{2 g_{1}}{\omega}\right) e^{\bar{a}_{1} \omega+G_{1}}, \bar{b}_{1} e^{\bar{a}_{1} \omega+G_{1}}, \bar{h}_{1}\right)=l_{1}^{+}, \\
0 & <H_{i}=l_{i}^{-} e^{-\left(\bar{c}_{i, i-1} \omega+G_{i}\right)}<l_{i}^{-}=g\left(\left(\bar{c}_{i, i-1}+\frac{2 g_{i}}{\omega}\right) e^{\bar{c}_{i, i-1} \omega+G_{i}}, \bar{b}_{i} e^{\bar{c}_{i, i-1} \omega+G_{i}}, \bar{h}_{i}\right) \\
& <g\left(\frac{2 g_{i}}{\omega}-\bar{d}_{i}-\bar{c}_{i, i+1} l_{i+1}^{+}, \bar{b}_{i} e^{\bar{c}_{i, i-1} \omega+G_{i}}, \bar{h}_{i}\right)=B_{i}^{-} \\
& <B_{i}^{+}=f\left(\frac{2 g_{i}}{\omega}-\bar{d}_{i}-\bar{c}_{i, i+1} l_{i+1}^{+}, \bar{b}_{i} e^{\bar{c}_{i, i-1} \omega+G_{i}}, \bar{h}_{i}\right) \\
& <f\left(\left(\bar{c}_{i, i-1}+\frac{2 g_{i}}{\omega}\right) e^{\bar{c}_{i, i-1} \omega+G_{i}}, \bar{b}_{i} e^{\bar{c}_{i, i-1} \omega+G_{i}}, \bar{h}_{i}\right)=l_{i}^{+}, \quad i=2,3, \ldots, n-1,
\end{aligned}
$$


ISRN Applied Mathematics

$$
\begin{aligned}
0 & <H_{n}=l_{n}^{-} e^{-\left(\bar{c}_{n, n-1} \omega+G_{n}\right)}<l_{n}^{-}=g\left(\left(\bar{c}_{n, n-1}+\frac{2 g_{n}}{\omega}\right) e^{\bar{c}_{n, n-1} \omega+G_{n}}, \bar{b}_{n} e^{\bar{c}_{n, n-1} \omega+G_{n}}, \bar{h}_{n}\right) \\
& <g\left(\frac{2 g n}{\omega}-\bar{d}_{n}, \bar{b}_{n} e^{\bar{c}_{n, n-1} \omega+G_{n}}, \bar{h}_{n}\right)=B_{n}^{-}<B_{n}^{+}=f\left(\frac{2 g_{n}}{\omega}-\bar{d}_{n}, \bar{b}_{n} e^{\bar{c}_{n, n-1} \omega+G_{n}}, \bar{h}_{n}\right) \\
& <f\left(\left(\bar{c}_{n, n-1}+\frac{2 g_{n}}{\omega}\right) e^{\bar{c}_{n, n-1} \omega+G_{n}}, \bar{b}_{n} e^{\bar{c}_{n, n-1} \omega+G_{n}}, \bar{h}_{n}\right)=l_{n}^{+}
\end{aligned}
$$

which imply that $\ln H_{i}<\ln l_{i}^{-}<\ln B_{i}^{-}<\ln B_{i}^{+}<\ln l_{i}^{+}, i=1,2, \ldots, n$; that is, the inequality (2) of Lemma 3.4 holds. Let us now to prove the inequality (3) of Lemma 3.4. In fact, since

$$
\begin{aligned}
& \max \left\{\ln A_{i}^{-}, \ln B_{i}^{-}\right\}=\frac{\ln A_{i}^{-}+\ln B_{i}^{-}+\left|\ln A_{i}^{-}-\ln B_{i}^{-}\right|}{2} \quad(1 \leq i \leq n), \\
& \min \left\{\ln A_{i}^{+}, \ln B_{i}^{+}\right\}=\frac{\ln A_{i}^{+}+\ln B_{i}^{+}-\left|\ln A_{i}^{+}-\ln B_{i}^{+}\right|}{2} \quad(1 \leq i \leq n),
\end{aligned}
$$

we have

$$
\begin{aligned}
\min & \left\{\ln A_{i}^{+}, \ln B_{i}^{+}\right\}-\max \left\{\ln A_{i}^{-}, \ln B_{i}^{-}\right\} \\
& =\frac{1}{2}\left[\ln A_{i}^{+}+\ln B_{i}^{+}-\ln A_{i}^{-}-\ln B_{i}^{-}-\left|\ln A_{i}^{-}-\ln B_{i}^{-}\right|-\left|\ln A_{i}^{+}-\ln B_{i}^{+}\right|\right] .
\end{aligned}
$$

If $A_{i}^{+} \geq B_{i}^{+}$, then $A_{i}^{-} \leq B_{i}^{-}$, and

$$
\min \left\{\ln A_{i}^{+}, \ln B_{i}^{+}\right\}-\max \left\{\ln A_{i}^{-}, \ln B_{i}^{-}\right\}=\ln B_{i}^{+}-\ln B_{i}^{-}>0 ;
$$

If $A_{i}^{+} \leq B_{i}^{+}$, then $A_{i}^{-} \geq B_{i}^{-}$, and

$$
\min \left\{\ln A_{i}^{+}, \ln B_{i}^{+}\right\}-\max \left\{\ln A_{i}^{-}, \ln B_{i}^{-}\right\}=\ln A_{i}^{+}-\ln A_{i}^{-}>0 .
$$

So, we drive $\min \left\{\ln A_{i}^{+}, \ln B_{i}^{+}\right\}>\max \left\{\ln A_{i}^{-}, \ln B_{i}^{-}\right\}$. From the above, we have completed the proof of Lemma 3.4.

Theorem 3.5. Assume that $\left(H_{1}\right),\left(H_{2}\right)$, and $\left(H_{3}\right)$ hold. Then system (1.3) has at least $2^{n}$ w-periodic solutions.

Proof. Let $C\left[I_{\omega} ; t_{1}, \ldots, t_{q}\right]=\left\{u: I_{\omega} \rightarrow \mathbb{R}^{n}\right.$ is a piecewise continuous map with first-class discontinuity points in $I_{\omega} \cap\left\{t_{k}\right\}_{k=1}^{\infty}$, and at each discontinuity point, it is continuous on the left\}. Take

$$
X=\left\{u=\left(u_{1}, u_{2}, \ldots, u_{n}\right)^{T} \in C\left[I_{\omega} ; t_{1}, \ldots, t_{q}\right]: u_{i}(t+\omega)=u_{i}(t)\right\}, \quad Z=X \times \mathbb{R}^{n \times(q+1)}
$$


and define

$$
\begin{gathered}
\|u\|_{0}=\sum_{i=1}^{n} \sup _{t \in \mathrm{I}_{\omega}}\left|u_{i}(t)\right|, \quad u \in X, \\
\|z\|_{1}=\|u\|_{0}+\sum_{j=1}^{q}\left|\xi_{j}\right|, \quad z=\left(u, \xi_{1}, \xi_{2}, \ldots, \xi_{q}\right) \in Z .
\end{gathered}
$$

Then both $\left(X,\|\cdot\|_{0}\right)$ and $\left(Z,\|\cdot\|_{1}\right)$ are Banach spaces. Let

$$
\begin{gathered}
\operatorname{Dom} L=\left\{u \in X: u^{\Delta} \in X\right\}, \quad L: \operatorname{Dom} L \longrightarrow Z, N: X \rightarrow Z, \\
L u=\left(\left(\begin{array}{c}
u_{1}^{\Delta} \\
u_{2}^{\Delta} \\
\vdots \\
u_{n}^{\Delta}
\end{array}\right),\left(\begin{array}{c}
\Delta u_{1}\left(t_{1}\right) \\
\Delta u_{2}\left(t_{1}\right) \\
\vdots \\
\Delta u_{n}\left(t_{1}\right)
\end{array}\right), \ldots,\left(\begin{array}{c}
\Delta u_{1}\left(t_{q}\right) \\
\Delta u_{2}\left(t_{q}\right) \\
\vdots \\
\Delta u_{n}\left(t_{q}\right)
\end{array}\right)\right), \\
N(u, \lambda)=\left(\left(\begin{array}{c}
F_{1}(t, \lambda) \\
\vdots \\
F_{i}(t, \lambda) \\
\vdots \\
F_{n}(t, \lambda)
\end{array}\right),\left(\begin{array}{c}
\ln \left(1+g_{11}\right) \\
\vdots \\
\ln \left(1+g_{i 1}\right) \\
\vdots \\
\ln \left(1+g_{n 1}\right)
\end{array}\right), \ldots,\left(\begin{array}{c}
\ln \left(1+g_{1 q}\right) \\
\vdots \\
\ln \left(1+g_{i q}\right) \\
\vdots \\
\ln \left(1+g_{n q}\right)
\end{array}\right)\right),
\end{gathered}
$$

where

$$
\begin{gathered}
F_{1}(t, \lambda)=a_{1}(t)-b_{1}(t) e^{u_{1}\left(t-\tau_{11}(t)\right)}-\lambda c_{12}(t) e^{u_{2}\left(t-\tau_{12}(t)\right)}-h_{1}(t) e^{-u_{1}(t)}, \\
F_{i}(t, \lambda)=-\lambda d_{i}(t)-b_{i}(t) e^{u_{i}\left(t-\tau_{i i}(t)\right)}+c_{i, i-1}(t) e^{u_{i-1}\left(t-\tau_{i, i-1}(t)\right)} \\
-\lambda c_{i, i+1}(t) e^{u_{i+1}\left(t-\tau_{i, i+1}(t)\right)}-h_{i}(t) e^{-u_{i}(t)}, \quad i=2,3, \ldots, n-1, \\
F_{n}(t, \lambda)=-\lambda d_{n}(t)-b_{n}(t) e^{u_{n}\left(t-\tau_{n n}(t)\right)}+c_{n, n-1}(t) e^{u_{n-1}\left(t-\tau_{n, n-1}(t)\right)}-h_{n}(t) e^{-u_{n}(t)} . \\
P: X \longrightarrow X, \quad Q: Z \longrightarrow Z, \\
P\left(u=\left(u_{1}, u_{2}, \ldots, u_{n}\right)^{T}\right)=\left(\bar{u}_{1}, \bar{u}_{2}, \ldots, \bar{u}_{n}\right)^{T}, \quad u \in X, \\
Q\left(\left(\begin{array}{c}
u_{1} \\
u_{2} \\
\vdots \\
u_{n}
\end{array}\right),\left\{\left(\begin{array}{c}
m_{1 k} \\
m_{2 k} \\
\vdots \\
m_{n k}
\end{array}\right)\right\}_{k=1}^{q}\right)=\left(\left(\begin{array}{c}
\bar{u}_{1}+\frac{1}{\omega} \sum_{k=1}^{q} m_{1 k} \\
\bar{u}_{2}+\frac{1}{\omega} \sum_{k=1}^{q} m_{2 k} \\
\vdots \\
\bar{u}_{n}+\frac{1}{\omega} \sum_{k=1}^{q} m_{n k}
\end{array}\right),\left\{\left(\begin{array}{c}
0 \\
0 \\
\vdots \\
0
\end{array}\right)\right\}_{k=1}^{q}\right) .
\end{gathered}
$$


Obviously,

$$
\begin{gathered}
\operatorname{Ker} L=\left\{u=\left(u_{1}, u_{2}, \ldots, u_{n}\right)^{T} \in X:\left(u_{1}(t), u_{2}(t), \ldots, u_{n}(t)\right)=\left(c_{1}, c_{2}, \ldots, c_{n}\right) \in \mathbb{R}^{n}, t \in \mathbb{T}\right\}, \\
\left.\operatorname{Im} L=\left\{\left(\begin{array}{c}
u_{1} \\
u_{2} \\
\vdots \\
u_{n}
\end{array}\right),\left\{\left(\begin{array}{c}
m_{1 k}+\sum_{k=1}^{q} m_{1 k}=0 \\
m_{2 k} \\
\vdots \\
m_{n k}
\end{array}\right)\right\}_{k=1}^{q}\right) \in Z \begin{array}{r}
\omega \bar{u}_{2}+\sum_{k=1}^{q} m_{2 k}=0 \\
\vdots \\
\\
\omega \bar{u}_{n}+\sum_{k=1}^{q} m_{n k}=0
\end{array}\right\} .
\end{gathered}
$$

Since $\operatorname{Im} L$ is closed in $Z, \operatorname{Im} P=\operatorname{Ker} L, \operatorname{Ker} Q=\operatorname{Im} L$, and $\operatorname{dim} \operatorname{Ker} L=n=\operatorname{codim} \operatorname{Im} L$, we know that $L$ is a Fredholm mapping of index zero. Furthermore, the generalized inverse (to L) $K_{P}: \operatorname{Im} L \rightarrow \operatorname{Ker} P \cap \operatorname{Dom} L$ is given by

$$
\begin{aligned}
K_{P}\left(z=\left(\left(\begin{array}{c}
u_{1} \\
u_{2} \\
\vdots \\
u_{n}
\end{array}\right),\left\{\left(\begin{array}{c}
m_{1 k} \\
m_{2 k} \\
\vdots \\
m_{n k}
\end{array}\right)\right\}_{k=1}^{q}\right)\right) \\
=\left(\begin{array}{l}
\int_{\kappa}^{t} u_{1}(s) \Delta s+\sum_{\kappa<t_{k}<t} m_{1 k}-\frac{1}{\omega} \sum_{k=1}^{q} m_{1 k}-\frac{1}{\omega} \int_{\kappa}^{\kappa+\omega} \int_{\kappa}^{t} u_{1}(s) \Delta s \Delta t \\
\int_{\kappa}^{t} u_{2}(s) \Delta s+\sum_{\kappa<t_{k}<t} m_{2 k}-\frac{1}{\omega} \sum_{k=1}^{q} m_{2 k}-\frac{1}{\omega} \int_{\kappa}^{\kappa+\omega} \int_{\kappa}^{t} u_{2}(s) \Delta s \Delta t \\
\vdots \\
\int_{\kappa}^{t} u_{1}(s) \Delta s+\sum_{\kappa<t_{k}<t} m_{1 k}-\frac{1}{\omega} \sum_{k=1}^{q} m_{n k}-\frac{1}{\omega} \int_{\kappa}^{\kappa+\omega} \int_{\kappa}^{t} u_{n}(s) \Delta s \Delta t
\end{array}\right) .
\end{aligned}
$$

Then

$$
Q N(u, \lambda)=\left(\left(\begin{array}{c}
\frac{1}{\omega} \int_{\kappa}^{\kappa+\omega} F_{1}(s, \lambda) \Delta s+\frac{2 g_{1}}{\omega} \\
\vdots \\
\frac{1}{\omega} \int_{\kappa}^{\kappa+\omega} F_{n}(s, \lambda) \Delta s+\frac{2 g_{n}}{\omega}
\end{array}\right),\left\{\left(\begin{array}{c}
0 \\
\vdots \\
0
\end{array}\right)\right\}_{k=1}^{q}\right)
$$




$$
\begin{aligned}
K_{P}(I-Q) N(u, \lambda)= & \left(\begin{array}{c}
\int_{\kappa}^{t} F_{1}(s, \lambda) \Delta s+\sum_{\kappa<t_{k}<t} \ln \left(1+g_{1 k}\right) \\
\vdots \\
\int_{\mathcal{K}}^{t} F_{n}(s, \lambda) \Delta s+\sum_{\kappa<t_{k}<t} \ln \left(1+g_{1 k}\right)
\end{array}\right) \\
& +\left(\frac{1}{2}-\frac{t}{\omega}\right)\left(\begin{array}{c}
\int_{\mathcal{\kappa}}^{\kappa+\omega} F_{1}(s, \lambda) \Delta s+2 g_{1} \\
\vdots \\
\int_{\mathcal{\kappa}}^{\kappa+\omega} F_{n}(s, \lambda) \Delta s+2 g_{n}
\end{array}\right) \\
& -\frac{1}{\omega}\left(\begin{array}{c}
\int_{\kappa}^{\kappa+\omega} \int_{\kappa}^{t} F_{1}(s, \lambda) \Delta s \Delta t+2 g_{1} \\
\int_{\kappa}^{\kappa+\omega} \int_{\kappa}^{t} F_{n}(s, \lambda) \Delta s \Delta t+2 g_{n}
\end{array}\right)
\end{aligned}
$$

where

$$
\begin{gathered}
F_{1}(s, \lambda)=a_{1}(s)-b_{1}(s) e^{u_{1}\left(s-\tau_{11}(s)\right)}-\lambda c_{12}(s) e^{u_{2}\left(s-\tau_{12}(s)\right)}-h_{1}(s) e^{-u_{1}(s)}, \\
F_{i}(s, \lambda)=-\lambda d_{i}(s)-b_{i}(s) e^{u_{i}\left(s-\tau_{i i}(s)\right)}+c_{i, i-1}(s) e^{u_{i-1}\left(s-\tau_{i, i-1}(s)\right)} \\
-\lambda c_{i, i+1}(s) e^{u_{i+1}\left(s-\tau_{i, i+1}(s)\right)}-h_{i}(s) e^{-u_{i}(s)}, \quad i=2,3, \ldots, n-1, \\
F_{n}(s, \lambda)=-\lambda d_{n}(s)-b_{n}(s) e^{u_{n}\left(s-\tau_{n n}(s)\right)}+c_{n, n-1}(s) e^{u_{n-1}\left(s-\tau_{n, n-1}(s)\right)}-h_{n}(s) e^{-u_{n}(s)} .
\end{gathered}
$$

By the Lebesgue convergence theorem, $Q N$ and $K_{P}(I-Q) N$ are continuous. Next, we show that $K_{P}(I-Q) N$ maps bounded sets into relatively compact sets. Let $\Omega \subset X$ be an arbitrary bounded set in $X$, then there exists a number $R>0$ such that $\|u\|<R$ for any $u \subset \Omega$. We prove that $\overline{K_{P}(I-Q) N(\bar{\Omega})}$ is relatively compact. In fact, for any $\left\{u_{v}=\left(u_{1}^{v}, u_{2}^{v}, \ldots, u_{n}^{v}\right)^{T}\right\}_{v \in \mathbb{N}} \in \Omega$ and $t \in I_{\omega}$, we have

$$
\begin{array}{r}
\left\|K_{P}(I-Q) N\left(u_{v}, \lambda\right)\right\|=\sum_{i=1}^{n} \sup _{t \in I_{\omega}} \mid \int_{\kappa}^{t} F_{i}(s, \lambda) \Delta s+\sum_{\kappa<t_{\kappa}<t} \ln \left(1+g_{i k}\right)+\left(\frac{1}{2}-\frac{t}{\omega}\right) \\
\quad \times \int_{\kappa}^{\kappa+\omega} F_{i}(s, \lambda) \Delta s+2 g_{i}-\frac{1}{\omega} \int_{\kappa}^{\kappa+\omega} \int_{\kappa}^{t} F_{i}(s, \lambda) \Delta s \Delta t+2 g_{1} \mid .
\end{array}
$$


Since $\left\|u_{v}\right\|<R$, it follows from the periodicity that there exists a constant $W_{1}$ such that $\left\|K_{P}(I-Q) N\left(u_{v}, \lambda\right)\right\| \leq W_{1}$. For any $\left\{u_{v}=\left(u_{1}^{v}, u_{2}^{v}, \ldots, u_{n}^{v}\right)^{T}\right\}_{v \in \mathbb{N}} \in \Omega$ and $t \in I_{\omega} \cap\left(t_{k}, t_{k+1}\right]$, $k=1,2, \ldots, q-1$, we have

$$
\left(K_{P}(I-Q) N\left(u_{v}, \lambda\right)\right)^{\Delta}=\left(\begin{array}{c}
F_{1}(s, \lambda) \\
\vdots \\
F_{n}(s, \lambda)
\end{array}\right)-\frac{1}{\omega}\left(\begin{array}{c}
\int_{\kappa}^{\kappa+\omega} F_{1}(s, \lambda) \Delta s+2 g_{1} \\
\vdots \\
\int_{\mathcal{K}}^{\kappa+\omega} F_{n}(s, \lambda) \Delta s+2 g_{n}
\end{array}\right) .
$$

Similarly, there has a constant $W_{2}$ such that

$$
\sum_{i=1}^{n} \sup _{t \in I_{\omega} \cap\left(t_{k}, t_{k+1}\right]}\left|\left(K_{P}(I-Q) N\left(u_{v}, \lambda\right)\right)^{\Delta}\right| \leq W_{2}, \quad k=1,2, \ldots, q-1 .
$$

It follows from the definition of $X$ that the right limit of $u_{v}(t)$ exists at each $t_{k}$ for $k=1,2, \ldots, q$. $v \in \mathbb{N}$. Without loss of generality, here, we can assume that $t_{1}=\mathcal{\kappa}$ and $t_{q}=\kappa+\omega$, for if $t_{1}>\mathcal{\kappa}$ or $t_{q}<\kappa+\omega$, we only need to add intervals $\left[\kappa, t_{1}\right] \cap \mathbb{T}$ or $\left[t_{q}, \kappa+\omega\right] \cap \mathbb{T}$ into the consideration. Consider the following functions:

$$
\begin{gathered}
\left(H\left(u_{v}, \lambda\right)\right)(t)= \begin{cases}\left(K_{P}(I-Q) N\left(u_{v}, \lambda\right)\right)(t), & t \in I_{\omega} \cap\left(t_{k}, t_{k+1}\right], k=1, \ldots, q-1, \\
\left(K_{P}(I-Q) N\left(u_{v}, \lambda\right)\right)\left(t_{k}+0\right), & t=t_{k}, k=1, \ldots, q,\end{cases} \\
\left(H\left(u_{v}, \lambda\right)\right)^{\Delta}(t)= \begin{cases}\left(K_{P}(I-Q) N\left(u_{v}, \lambda\right)\right)^{\Delta}(t), & t \in I_{\omega} \cap\left(t_{k}, t_{k+1}\right], k=1, \ldots, q-1, \\
\left(K_{P}(I-Q) N\left(u_{v}, \lambda\right)\right)^{\Delta}\left(t_{k}+0\right), & t=t_{k}, k=1, \ldots, q,\end{cases}
\end{gathered}
$$

where $v \in \mathbb{N}$. Then on each interval $\left[t_{k}, t_{k+1}\right],\left\{\left(H_{k} u_{v}\right)=\left.\left(H u_{v}\right)\right|_{\left[t_{k}, t_{k+1}\right]}\right\}_{v \in \mathbb{N}}$, and $\left\{\left(H_{k} u_{v}\right)^{\Delta}=\right.$ $\left.\left.\left(H u_{v}\right)^{\Delta}\right|_{\left[t_{k}, t_{k+1}\right]}\right\}_{v \in \mathbb{N}}$ are uniformly bounded on $\left[t_{k}, t_{k+1}\right] \cap \mathbb{T}, k=1,2, \ldots, q-1$. By Lemma 2.7, there exists a subsequence $\left\{H_{1} u_{v_{1}^{(1)}}\right\}_{v_{1}^{(1)} \in \mathbb{N}}$ of $\left\{H_{1} u_{v}\right\}_{v \in \mathbb{N}}$ converges uniformly on $\left[t_{1}, t_{2}\right] \cap$ $\mathbb{T}$. Similarly, there is a subsequence $\left\{H_{2} u_{v_{2}^{(2)}}\right\}_{v_{2}^{(2)} \in \mathbb{N}}$ of $\left\{H_{1} u_{v_{1}^{(1)}}\right\}_{v_{1}^{(1)} \in \mathbb{N}}$ converges uniformly on $\left[t_{2}, t_{3}\right] \cap \mathbb{T}$. Repeating such a process, we can obtain that there is a subsequence $\left\{H_{q-1} u_{v_{q-1}^{(q-1)}}\right\}_{v_{q-1}^{(q-1)} \in \mathbb{N}}$ of $\left\{H_{q-2} u_{v_{q-2}^{(q-2)}}\right\}_{v_{q-2}^{(q-2)} \in \mathbb{N}}$ converges uniformly on $\left[t_{q-1}, t_{q}\right] \cap \mathbb{T}$. Obviously, $\left\{H_{q-1} u_{v_{q-1}^{(q-1)}}\right\}_{v_{q-1}^{(q-1)} \in \mathbb{N}}$ is a subsequence of $\left\{H u_{v}\right\}_{v \in \mathbb{N}}$ that converges uniformly on $I_{\omega}=[\kappa, \kappa+$ $\omega] \cap \mathbb{T}$. Therefore, $\left\{K_{P}(I-Q) N_{q-1} u_{v_{q-1}^{(q-1)}}\right\}_{v_{q-1}^{(q-1)} \in \mathbb{N}}$ is a subsequence of $\left\{K_{P}(I-Q) N u_{v}\right\}_{v \in \mathbb{N}}$ that converges uniformly on $I_{\omega}$, namely, $\overline{K_{P}(I-Q) N(\bar{\Omega})}$ is relatively compact. Moreover, $Q N(\bar{\Omega})$ is bounded. Thus, $N$ is $L$-compact on $\bar{\Omega}$ for any open bounded set $\Omega \subset X$. 
In order to use Lemma 3.1, we have to find at least $2^{n}$ appropriate open bounded subsets of $X$. Considering the operator equation $L u=\lambda N(u, \lambda), \lambda \in(0,1)$, we have

$$
\begin{aligned}
& u_{1}^{\Delta}(t)=\lambda\left(a_{1}(t)-b_{1}(t) e^{u_{1}\left(t-\tau_{11}(t)\right)}-\lambda c_{12}(t) e^{u_{2}\left(t-\tau_{12}(t)\right)}-h_{1}(t) e^{-u_{1}(t)}\right), \\
& u_{i}^{\Delta}(t)=\lambda\left(-\lambda d_{i}(t)-b_{i}(t) e^{u_{i}\left(t-\tau_{i i}(t)\right)}+c_{i, i-1}(t) e^{u_{i-1}\left(t-\tau_{i, i-1}(t)\right)}-\lambda c_{i, i+1}(t) e^{u_{i+1}\left(t-\tau_{i, i+1}\right)}-h_{i}(t) e^{-u_{i}(t)}\right), \\
& 2 \leq i \leq n-1, \\
& u_{n}^{\Delta}(t)=\lambda\left(-\lambda d_{n}(t)-b_{n}(t) e^{u_{n}\left(t-\tau_{n n}(t)\right)}+c_{n, n-1}(t) e^{u_{n-1}\left(t-\tau_{n,-1}(t)\right)}-h_{n}(t) e^{-u_{n}(t)}\right), \\
& \Delta u_{j}\left(t_{k}\right)=\lambda \ln \left(1+g_{j k}\right), \quad 1 \leq j \leq n .
\end{aligned}
$$

Assume that $u \in X$ is an $\omega$-periodic solution of system (3.27) for some $\lambda \in(0,1)$. Integrating (3.27) from $\kappa$ to $\kappa+\omega$, we have

$$
\begin{gathered}
-2 g_{1}=\int_{\kappa}^{\kappa+\omega}\left\{a_{1}(t)-b_{1}(t) e^{u_{1}\left(t-\tau_{11}(t)\right)}-\lambda c_{12}(t) e^{u_{2}\left(t-\tau_{12}(t)\right)}-h_{1}(t) e^{-u_{1}(t)}\right\} \Delta t, \\
\vdots \\
-2 g_{i}=\int_{\kappa}^{\kappa+\omega}\left\{-\lambda d_{i}(t)-b_{i}(t) e^{u_{i}\left(t-\tau_{i i}(t)\right)}+c_{i, i-1}(t) e^{u_{i-1}\left(t-\tau_{i, i-1}(t)\right)}\right. \\
\left.\quad-\lambda c_{i, i+1}(t) e^{u_{i+1}\left(t-\tau_{i, i+1}(t)\right)}-h_{i}(t) e^{-u_{i}(t)}\right\} \Delta t, \quad 2 \leq i \leq n-1, \\
\quad \vdots \quad \\
-2 g_{n}=\int_{\kappa}^{\kappa+\omega}\left\{-\lambda d_{n}(t)-b_{n}(t) e^{u_{n}\left(t-\tau_{n n}(t)\right)}+c_{n, n-1}(t) e^{u_{n-1}\left(t-\tau_{n, n-1}(t)\right)}-h_{n}(t) e^{-u_{n}(t)}\right\} \Delta t .
\end{gathered}
$$

Then

$$
\int_{\mathcal{K}}^{\mathcal{K}+\omega}\left\{b_{1}(t) e^{u_{1}\left(t-\tau_{11}(t)\right)}+\lambda c_{12}(t) e^{u_{2}\left(t-\tau_{12}(t)\right)}+h_{1}(t) e^{-u_{1}(t)}\right\} \Delta t=\bar{a}_{1} \omega+2 g_{1}
$$




$$
\begin{aligned}
& \int_{\kappa}^{\kappa+\omega}\left\{\lambda d_{i}(t)+b_{i}(t) e^{u_{i}\left(t-\tau_{i i}(t)\right)}+\lambda c_{i, i+1}(t) e^{u_{i+1}\left(t-\tau_{i, i+1}(t)\right)}+h_{i}(t) e^{-u_{i}(t)}\right\} \Delta t \\
& \quad=\int_{\mathcal{\kappa}}^{\kappa+\omega} c_{i, i-1}(t) e^{u_{i-1}\left(t-\tau_{i, i-1}(t)\right)} \Delta t+2 g_{i}, \quad 2 \leq i \leq n-1, \\
& \quad \vdots \\
& \int_{\kappa}^{\kappa+\omega}\left\{\lambda d_{n}(t)+b_{n}(t) e^{u_{n}\left(t-\tau_{n n}(t)\right)}+h_{n}(t) e^{-u_{n}(t)}\right\} \Delta t=\int_{\kappa}^{\kappa+\omega} c_{n, n-1}(t) e^{u_{n-1}\left(t-\tau_{n, n-1}(t)\right)} \Delta t+2 g_{n} .
\end{aligned}
$$

Furthermore, Note that $u=\left(u_{1}, u_{2}, \ldots, u_{n}\right)^{T} \in X$, and there exists $\xi_{i}, \eta_{i} \in[\kappa, \kappa+\omega] \cap \mathbb{T}=I_{\omega}$, $i=1,2, \ldots, n$, such that $u_{i}\left(\xi_{i}\right)=\sup _{t \in I_{\omega}} u_{i}(t), u_{i}\left(\eta_{i}\right)=\inf _{t \in I_{\omega}} u_{i}(t)$.

On the one hand, according to the first equation of (3.27) and (3.29), we have

$$
\int_{\kappa}^{\kappa+\omega}\left|u_{1}^{\Delta}(t)\right| \Delta t<2 \bar{a}_{1} \omega+2 g_{1}
$$

In the light of the first of equation of (3.29), (3.30), and Lemma 3.2, we get

$$
\begin{aligned}
\omega \bar{b}_{1} e^{u_{1}\left(\grave{\xi}_{1}\right)-\left(\bar{a}_{1} \omega+G_{1}\right)}+\omega \bar{h}_{1} e^{-u_{1}\left(\xi_{1}\right)} & \leq \omega \bar{b}_{1} e^{u_{1}\left(\xi_{1}\right)-(1 / 2)\left[\int_{\kappa}^{\kappa+\omega}\left|u_{1}^{\Delta}(s)\right| \Delta s+\sum_{k=1}^{q}\left|\Delta u_{1}\left(t_{k}\right)\right|\right]}+\omega \bar{h}_{1} e^{-u_{1}\left(\xi_{1}\right)} \\
& <\omega \bar{b}_{1} e^{u_{1}\left(\eta_{1}\right)}+\omega \bar{h}_{1} e^{-u_{1}\left(\xi_{1}\right)} \\
& <\int_{\kappa}^{\kappa+\omega}\left\{b_{1}(t) e^{u_{1}\left(t-\tau_{11}(t)\right)}+\lambda c_{12}(t) e^{u_{2}\left(t-\tau_{12}(t)\right)}+h_{1}(t) e^{-u_{1}(t)}\right\} \Delta t \\
& =\bar{a}_{1} \omega+2 g_{1},
\end{aligned}
$$

namely,

$$
\bar{b}_{1} e^{2 u_{1}\left(\xi_{1}\right)}-\left(\bar{a}_{1}+\frac{2 g_{1}}{\omega}\right) e^{\bar{a}_{1} \omega+G_{1}} e^{u_{1}\left(\xi_{1}\right)}+\bar{h}_{1} e^{\bar{a}_{1} \omega+G_{1}}<0,
$$

which implies that

$$
\ln l_{1}^{-}<u_{1}\left(\xi_{1}\right)<\ln l_{1}^{+}
$$

Applying Lemma 3.2, we drive

$$
\ln H_{1}=\ln l_{1}^{-}-\left(\bar{a}_{1} \omega+G_{1}\right)<u_{1}(\eta) \leq u_{1}\left(\xi_{1}\right)<\ln l_{1}^{+} .
$$


According to (3.29) and (3.30), we get

$$
\int_{\kappa}^{\kappa+\omega}\left|u_{2}^{\Delta}(t)\right| \Delta t<2 \bar{c}_{21} \omega+2 g_{2}
$$

By (3.29) and (3.35), we have

$$
\begin{aligned}
& \omega \bar{b}_{2} e^{u_{2}\left(\xi_{2}\right)-\left(\bar{c}_{21} \omega+G_{2}\right)}+\omega \bar{h}_{2} e^{-u_{2}\left(\xi_{2}\right)} \\
& \leq \omega \bar{b}_{2} e^{u_{2}\left(\xi_{2}\right)-(1 / 2)\left[\int_{\kappa}^{\kappa+\omega}\left|u_{2}^{\Delta}(s)\right| \Delta s+\sum_{k=1}^{q}\left|\Delta u_{2}\left(t_{k}\right)\right|\right]}+\omega \bar{h}_{2} e^{-u_{2}\left(\xi_{2}\right)}<\omega \bar{b}_{2} e^{u_{2}\left(\eta_{2}\right)}+\omega \bar{h}_{2} e^{-u_{2}\left(\xi_{2}\right)} \\
& \quad<\int_{\kappa}^{\kappa+\omega}\left\{\lambda d_{2}(t)+b_{2}(t) e^{u_{2}\left(t-\tau_{22}(t)\right)}+\lambda c_{23}(t) e^{u_{3}\left(t-\tau_{23}(t)\right)}+h_{2}(t) e^{-u_{2}(t)}\right\} \Delta t \\
& \quad=\int_{\kappa}^{\kappa+\omega} c_{21}(t) e^{u_{1}\left(t-\tau_{21}(t)\right)} \Delta t+2 g_{1}<\omega \bar{c}_{21} l_{1}^{+}+2 g_{2},
\end{aligned}
$$

that is

$$
\bar{b}_{2} e^{2 u_{2}\left(\xi_{2}\right)}-\left(\bar{c}_{21}+\frac{2 g_{2}}{\omega}\right) e^{\bar{c}_{21} \omega+G_{2}} e^{u_{2}\left(\xi_{2}\right)}+\bar{h}_{2} e^{\bar{c}_{21} \omega+G_{2}}<0,
$$

which implies that

$$
\ln l_{2}^{-}<u_{2}\left(\xi_{2}\right)<\ln l_{2}^{+}
$$

Applying Lemma 3.2, we drive

$$
\ln H_{2}=\ln l_{2}^{-}-\left(\bar{c}_{21} \omega+G_{2}\right)<u_{2}(\eta) \leq u_{2}\left(\xi_{2}\right)<\ln l_{2}^{+} .
$$

By deducing for $i=3,4, \ldots, n$, we obtain

$$
\begin{gathered}
\int_{\kappa}^{\kappa+\omega}\left|u_{i}^{\Delta}(t)\right| \Delta t<2 \bar{c}_{i, i-1} \omega+2 g_{i}, \\
\omega \bar{b}_{i} e^{u_{i}\left(\bar{\xi}_{i}\right)-\left(\bar{c}_{i, i-1} \omega+G_{i}\right)}+\omega \bar{h}_{i} e^{-u_{i}\left(\xi_{i}\right)} \\
\leq \omega \bar{b}_{i} e^{u_{i}\left(\xi_{i}\right)-(1 / 2)\left[\int_{\kappa}^{\kappa+\omega}\left|u_{i}^{\Delta}(s)\right| \Delta s+\sum_{k=1}^{q}\left|\Delta u_{i}\left(t_{k}\right)\right|\right]}+\omega \bar{h}_{i} e^{-u_{i}\left(\xi_{i}\right)}<\omega \bar{b}_{i} e^{u_{i}\left(\eta_{i}\right)}+\omega \bar{h}_{i} e^{-u_{i}\left(\xi_{i}\right)} \\
<\int_{\kappa}^{\kappa+\omega}\left\{\lambda d_{i}(t)+b_{i}(t) e^{u_{i}\left(t-\tau_{i i}(t)\right)}+\lambda c_{i, i+1}(t) e^{u_{i+1}\left(t-\tau_{i, i+1}(t)\right)}+h_{i}(t) e^{-u_{i}(t)}\right\} \Delta t \\
=\int_{\kappa}^{\kappa+\omega} c_{i, i-1}(t) e^{u_{i-1}\left(t-\tau_{i, i-1}(t)\right)} \Delta t+2 g_{i-1}<\omega \bar{c}_{i, i-1} l_{i-1}^{+}+2 g_{i}
\end{gathered}
$$


namely,

$$
\bar{b}_{i} e^{2 u_{i}\left(\xi_{i}\right)}-\left(\bar{c}_{i, i-1}+\frac{2 g_{i}}{\omega}\right) e^{\bar{c}_{i, i-1} \omega+G_{i}} e^{u_{i}\left(\xi_{i}\right)}+\bar{h}_{i} e^{\bar{c}_{i, i-1} \omega+G_{i}}<0,
$$

which implies that

$$
\ln l_{i}^{-}<u_{i}\left(\xi_{i}\right)<\ln l_{i}^{+}, \quad i=3,4, \ldots, n .
$$

Applying Lemma 3.2, we drive

$$
\ln H_{i}=\ln l_{i}^{-}-\left(\bar{c}_{i, i-1} \omega+G_{i}\right)<u_{i}(\eta) \leq u_{i}\left(\xi_{i}\right)<\ln l_{i}^{+}, \quad i=3,4, \ldots, n .
$$

On the other hand, in the light of the first equation of (3.29) and (3.30), we have

$$
\begin{aligned}
\bar{a}_{1} \omega+2 g_{1} & =\int_{\kappa}^{\kappa+\omega}\left\{b_{1}(t) e^{u_{1}\left(t-\tau_{11}(t)\right)}+\lambda c_{12}(t) e^{u_{2}\left(t-\tau_{12}(t)\right)}+h_{1}(t) e^{-u_{1}(t)}\right\} \Delta t \\
& <\bar{b}_{1} \omega e^{u_{1}\left(\xi_{1}\right)}+\bar{c}_{12} \omega l_{2}^{+}+\bar{h}_{1} \omega e^{-u_{1}\left(\eta_{1}\right)} \\
& <\bar{b}_{1} \omega e^{u_{1}\left(\xi_{1}\right)}+\bar{c}_{12} \omega l_{2}^{+}+\bar{h}_{1} \omega e^{-u_{1}\left(\xi_{1}\right)+(1 / 2)\left[\int_{\kappa}^{\kappa+\omega}\left|u_{1}^{\Delta}(s)\right| \Delta s+\sum_{k=1}^{q}\left|\Delta u_{1}\left(t_{k}\right)\right|\right]} \\
& <\bar{b}_{1} \omega e^{u_{1}\left(\xi_{1}\right)}+\bar{c}_{12} \omega l_{2}^{+}+\bar{h}_{1} \omega e^{-u_{1}\left(\xi_{1}\right)+a_{1} \omega+G_{1}},
\end{aligned}
$$

namely,

$$
\bar{b}_{1} e^{2 u_{1}\left(\xi_{1}\right)}-\left(\bar{a}_{1}+\frac{2 g_{1}}{\omega}-\bar{c}_{12} l_{2}^{+}\right) e^{u_{1}\left(\xi_{1}\right)}+\bar{h}_{1} e^{\bar{a}_{1} \omega+G_{1}}>0,
$$

which implies that

$$
\begin{aligned}
\ln A_{1}^{+}<u_{1}\left(\xi_{1}\right) \quad \text { or } \quad u_{1}\left(\xi_{1}\right)<\ln A_{1}^{-}, \\
\bar{a}_{1} \omega+2 g_{1}=\int_{\kappa}^{\kappa+\omega}\left\{b_{1}(t) e^{u_{1}\left(t-\tau_{11}(t)\right)}+\lambda c_{12}(t) e^{u_{2}\left(t-\tau_{12}(t)\right)}+h_{1}(t) e^{-u_{1}(t)}\right\} \Delta t \\
<\omega\left(\bar{b}_{1} e^{u_{1}\left(\xi_{1}\right)}+\bar{c}_{12} l_{2}^{+}+\bar{h}_{1} e^{-u_{1}\left(\eta_{1}\right)}\right) \\
<\omega\left(\bar{b}_{1} e^{u_{1}\left(\eta_{1}\right)+(1 / 2)\left[\int_{\kappa}^{\kappa+\omega}\left|u_{1}^{\Delta}(s)\right| \Delta s+\sum_{k=1}^{q}\left|\Delta u_{1}\left(t_{k}\right)\right|\right]}+\bar{c}_{12} l_{2}^{+}+\bar{h}_{1} e^{-u_{1}\left(\eta_{1}\right)}\right) \\
<\bar{b}_{1} \omega e^{u_{1}\left(\eta_{1}\right)+\bar{a}_{1} \omega+G_{1}}+\bar{c}_{12} \omega l_{2}^{+}+\bar{h}_{1} \omega e^{-u_{1}\left(\eta_{1}\right)},
\end{aligned}
$$

that is,

$$
\bar{b}_{1} e^{2 u_{1}\left(\eta_{1}\right)}-\left(\bar{a}_{1}+\frac{2 g_{1}}{\omega}-\bar{c}_{12} l_{2}^{+}\right) e^{-\left(\bar{a}_{1} \omega+G_{1}\right)} e^{u_{1}\left(\eta_{1}\right)}+\bar{h}_{1} e^{-\left(\bar{a}_{1} \omega+G_{1}\right)}>0,
$$


which implies that

$$
\ln B_{1}^{+}<u_{1}\left(\eta_{1}\right) \quad \text { or } \quad u_{1}\left(\eta_{1}\right)<\ln B_{1}^{-} \text {. }
$$

By (3.29) and (3.35), we have

$$
\begin{aligned}
2 g_{2} & <\int_{\kappa}^{\kappa+\omega} c_{21}(t) e^{u_{1}\left(t-\tau_{21}(t)\right)} \Delta t+2 g_{2} \\
& =\int_{\kappa}^{\kappa+\omega}\left\{\lambda d_{2}(t)+b_{2}(t) e^{u_{2}\left(t-\tau_{22}(t)\right)}+\lambda c_{23}(t) e^{u_{3}\left(t-\tau_{23}(t)\right)}+h_{2}(t) e^{-u_{2}(t)}\right\} \Delta t \\
& <\bar{b}_{2} \omega e^{u_{2}\left(\xi_{2}\right)}+\left(\bar{d}_{2}+\bar{c}_{23} l_{3}^{+}\right) \omega+\bar{h}_{2} \omega e^{-u_{2}\left(\eta_{2}\right)} \\
& <\bar{b}_{2} \omega e^{u_{2}\left(\xi_{2}\right)}+\left(\bar{d}_{2}+\bar{c}_{23} l_{3}^{+}\right) \omega+\bar{h}_{2} \omega e^{-u_{2}\left(\xi_{2}\right)+(1 / 2)\left[\int_{\kappa}^{\kappa+\omega}\left|u_{2}^{\Delta}(s)\right| \Delta s+\sum_{k=1}^{q}\left|\Delta u_{2}\left(t_{k}\right)\right|\right]} \\
& <\bar{b}_{2} \omega e^{u_{2}\left(\xi_{2}\right)}+\left(\bar{d}_{2}+\bar{c}_{23} l_{3}^{+}\right) \omega+\bar{h}_{2} \omega e^{-u_{2}\left(\xi_{2}\right)+\bar{c}_{21} \omega+G_{2}},
\end{aligned}
$$

namely,

$$
\bar{b}_{2} e^{2 u_{2}\left(\xi_{2}\right)}-\left(\frac{2 g_{2}}{\omega}-\bar{d}_{2}-\bar{c}_{23} l_{3}^{+}\right) e^{u_{2}\left(\xi_{2}\right)}+\bar{h}_{2} e^{\bar{c}_{21} \omega+G_{2}}>0,
$$

which implies that

$$
\begin{aligned}
& \ln A_{2}^{+}<u_{2}\left(\xi_{2}\right) \quad \text { or } \quad u_{2}\left(\xi_{2}\right)<\ln A_{2}^{-} \text {, } \\
& 2 g_{2}<\int_{\kappa}^{\kappa+\omega} c_{21}(t) e^{u_{1}\left(t-\tau_{21}(t)\right)} \Delta t+2 g_{2} \\
& =\int_{\kappa}^{\kappa+\omega}\left\{\lambda d_{2}(t)+b_{2}(t) e^{u_{2}\left(t-\tau_{22}(t)\right)}+\lambda c_{23}(t) e^{u_{3}\left(t-\tau_{23}(t)\right)}+h_{2}(t) e^{-u_{2}(t)}\right\} \Delta t \\
& <\bar{b}_{2} \omega e^{u_{2}\left(\xi_{2}\right)}+\left(\bar{d}_{2}+\bar{c}_{23} l_{3}^{+}\right) \omega+\bar{h}_{2} \omega e^{-u_{2}\left(\eta_{2}\right)} \\
& <\bar{b}_{2} \omega e^{u_{2}\left(\eta_{2}\right)+(1 / 2)\left[\int_{\kappa}^{\kappa+\omega}\left|u_{2}^{\Delta}(s)\right| \Delta s+\sum_{k=1}^{q}\left|\Delta u_{2}\left(t_{k}\right)\right|\right]}+\left(\bar{d}_{2}+\bar{c}_{23} l_{3}^{+}\right) \omega+\bar{h}_{2} \omega e^{-u_{2}\left(\eta_{2}\right)} \\
& <\bar{b}_{2} \omega e^{u_{2}\left(\eta_{2}\right)+\bar{c}_{21} \omega+G_{2}}+\left(\bar{d}_{2}+\bar{c}_{23} l_{3}^{+}\right) \omega+\bar{h}_{2} \omega e^{-u_{2}\left(\eta_{2}\right)},
\end{aligned}
$$

that is,

$$
\bar{b}_{2} e^{2 u_{2}\left(\eta_{2}\right)}-\left(\frac{2 g_{2}}{\omega}-\bar{d}_{2}-\bar{c}_{23} l_{3}^{+}\right) e^{-\left(\bar{c}_{21} \omega+G_{2}\right)} e^{u_{2}\left(\eta_{2}\right)}+\bar{h}_{2} e^{-\left(\bar{c}_{21} \omega+G_{2}\right)}>0,
$$

which implies that

$$
\ln B_{2}^{+}<u_{2}\left(\eta_{2}\right) \quad \text { or } \quad u_{2}\left(\eta_{2}\right)<\ln B_{2}^{-} \text {. }
$$


By deducing for $i=3,4, \ldots, n-1$, we obtain

$$
\begin{aligned}
2 g_{i} & <\int_{\mathcal{K}}^{\mathcal{\kappa}+\omega} c_{i, i-1}(t) e^{u_{i-1}\left(t-\tau_{i, i-1}(t)\right)} \Delta t+2 g_{i} \\
& =\int_{\mathcal{K}}^{\mathcal{\kappa}+\omega}\left\{\lambda d_{i}(t)+b_{i}(t) e^{u_{i}\left(t-\tau_{i i}(t)\right)}+\lambda c_{i, i+1}(t) e^{u_{i+1}\left(t-\tau_{i, 1}(t)\right)}+h_{i}(t) e^{-u_{i}(t)}\right\} \Delta t \\
& <\bar{b}_{i} \omega e^{u_{i}\left(\bar{\xi}_{i}\right)}+\left(\bar{d}_{i}+\bar{c}_{i, i+1} l_{i+1}^{+}\right) \omega+\bar{h}_{i} \omega e^{-u_{i}\left(\eta_{i}\right)} \\
& <\bar{b}_{i} \omega e^{u_{i}\left(\xi_{i}\right)}+\left(\bar{d}_{i}+\bar{c}_{i, i+1} l_{i+1}^{+}\right) \omega+\bar{h}_{i} \omega e^{-u_{i}\left(\xi_{i}\right)+(1 / 2)\left[\int_{\kappa}^{\kappa+\omega}\left|u_{i}^{\Delta}(s)\right| \Delta s+\sum_{k=1}^{q}\left|\Delta u_{i}\left(t_{k}\right)\right|\right]} \\
& <\bar{b}_{i} \omega e^{u_{i}\left(\bar{\xi}_{i}\right)}+\left(\bar{d}_{i}+\bar{c}_{i, i+1} l_{i+1}^{+}\right) \omega+\bar{h}_{i} \omega e^{-u_{i}\left(\bar{\xi}_{i}\right)+\bar{c}_{i, i-1} \omega+G_{i}},
\end{aligned}
$$

namely,

$$
\bar{b}_{i} e^{2 u_{i}\left(\xi_{i}\right)}-\left(\frac{2 g_{i}}{\omega}-\bar{d}_{i}-\bar{c}_{i, i+1} l_{i+1}^{+}\right) e^{u_{i}\left(\xi_{i}\right)}+\bar{h}_{i} e^{\bar{c}_{i, i-1} \omega+G_{i}}>0
$$

which implies that

$$
\begin{gathered}
\ln A_{i}^{+}<u_{i}\left(\xi_{i}\right) \quad \text { or } \quad u_{i}\left(\xi_{i}\right)<\ln A_{i}^{-}, \\
2 g_{i}<\int_{\kappa}^{\kappa+\omega} c_{i, i-1}(t) e^{u_{1}\left(t-\tau_{i, 1}(t)\right)} \Delta t+2 g_{i} \\
=\int_{\kappa}^{\kappa+\omega}\left\{\lambda d_{i}(t)+b_{i}(t) e^{u_{i}\left(t-\tau_{i i}(t)\right)}+\lambda c_{i, i+1}(t) e^{u_{i+1}\left(t-\tau_{i, i+1}(t)\right)}+h_{i}(t) e^{-u_{i}(t)}\right\} \Delta t \\
<\bar{b}_{i} \omega e^{u_{i}\left(\bar{\xi}_{i}\right)}+\left(\bar{d}_{i}+\bar{c}_{i, i+1} l_{i+1}^{+}\right) \omega+\bar{h}_{2} \omega e^{-u_{i}\left(\eta_{i}\right)} \\
<\bar{b}_{i} \omega e^{u_{i}\left(\eta_{i}\right)+(1 / 2)\left[\int_{\kappa}^{\kappa+\omega}\left|u_{i}^{\Delta}(s)\right| \Delta s+\sum_{k=1}^{q}\left|\Delta u_{i}\left(t_{k}\right)\right|\right]}+\left(\bar{d}_{i}+\bar{c}_{i, i+1} l_{i+1}^{+}\right) \omega+\bar{h}_{i} \omega e^{-u_{i}\left(\eta_{i}\right)} \\
<\bar{b}_{i} \omega e^{u_{i}\left(\eta_{i}\right)+\bar{c}_{i, i-1} \omega+G_{i}}+\left(\bar{d}_{i}+\bar{c}_{i, i+1} l_{i+1}^{+}\right) \omega+\bar{h}_{i} \omega e^{-u_{i}\left(\eta_{i}\right)},
\end{gathered}
$$

that is,

$$
\bar{b}_{i} e^{2 u_{i}\left(\eta_{i}\right)}-\left(\frac{2 g_{i}}{\omega}-\bar{d}_{i}-\bar{c}_{i, i+1} l_{i+1}^{+}\right) e^{-\left(\bar{c}_{i, i-1} \omega+G_{i}\right)} e^{u_{i}\left(\eta_{i}\right)}+\bar{h}_{i} e^{-\left(\bar{c}_{i, i-1} \omega+G_{i}\right)}>0,
$$

which implies that

$$
\ln B_{i}^{+}<u_{i}\left(\eta_{i}\right) \quad \text { or } \quad u_{i}\left(\eta_{i}\right)<\ln B_{i}^{-} .
$$


By the final equation of (3.29), we get

$$
\begin{aligned}
2 g_{n} & <\int_{\kappa}^{\kappa+\omega} c_{n, n-1}(t) e^{u_{n-1}\left(t-\tau_{n, n-1}(t)\right)} \Delta t+2 g_{n} \\
& =\int_{\kappa}^{\kappa+\omega}\left\{\lambda d_{n}(t)+b_{n}(t) e^{u_{n}\left(t-\tau_{n n}(t)\right)}+h_{n}(t) e^{-u_{n}(t)}\right\} \Delta t<\bar{d}_{n} \omega+\bar{b}_{n} \omega e^{u_{n}\left(\xi_{n}\right)}+\bar{h}_{n} \omega e^{-u_{n}\left(\eta_{n}\right)} \\
& <\bar{d}_{n} \omega+\bar{b}_{n} \omega e^{u_{n}\left(\xi_{n}\right)}+\bar{h}_{n} \omega e^{-u_{n}\left(\xi_{n}\right)+(1 / 2)\left[\int_{\kappa}^{\kappa+\omega}\left|u_{n}^{\Delta}(s)\right| \Delta s+\sum_{k=1}^{q}\left|\Delta u_{n}\left(t_{k}\right)\right|\right]} \\
& <\bar{d}_{n} \omega+\bar{b}_{n} \omega e^{u_{n}\left(\xi_{n}\right)}+\bar{h}_{n} \omega e^{-u_{n}\left(\xi_{n}\right)+\bar{c}_{n, n-1} \omega+G_{n}}
\end{aligned}
$$

namely,

$$
\bar{b}_{n} e^{2 u_{n}\left(\xi_{n}\right)}-\left(\frac{2 g_{n}}{\omega}-\bar{d}_{n}\right) e^{u_{n}\left(\xi_{n}\right)}+\bar{h}_{n} e^{\bar{c}_{n, n-1} \omega+G_{n}}>0
$$

which implies that

$$
\begin{aligned}
\ln A_{n}^{+}<u_{n}\left(\xi_{n}\right) \quad \text { or } \quad u_{n}\left(\xi_{n}\right)<\ln A_{n}^{-} \\
2 g_{n}<\int_{\kappa}^{\kappa+\omega} c_{n, n-1}(t) e^{u_{n-1}\left(t-\tau_{n, n-1}(t)\right)} \Delta t+2 g_{n} \\
=\int_{\kappa}^{\kappa+\omega}\left\{\lambda d_{n}(t)+b_{n}(t) e^{u_{n}\left(t-\tau_{n n}(t)\right)}+h_{n}(t) e^{-u_{n}(t)}\right\} \Delta t<\bar{d}_{n} \omega+\bar{b}_{n} \omega e^{u_{n}\left(\xi_{n}\right)}+\bar{h}_{n} \omega e^{-u_{n}\left(\eta_{n}\right)} \\
<\bar{d}_{n} \omega+\bar{b}_{n} \omega e^{u_{n}\left(\eta_{n}\right)+(1 / 2)\left[\int_{\kappa}^{\kappa+\omega}\left|u_{n}^{\Delta}(s)\right| \Delta s+\sum_{k=1}^{q}\left|\Delta u_{n}\left(t_{k}\right)\right|\right]}+\bar{h}_{n} \omega e^{-u_{n}\left(\eta_{n}\right)} \\
<\bar{d}_{n} \omega+\bar{b}_{n} \omega e^{u_{n}\left(\eta_{n}\right)+\bar{c}_{n, n-1} \omega+G_{n}}+\bar{h}_{n} \omega e^{-u_{n}\left(\eta_{n}\right)}
\end{aligned}
$$

namely,

$$
\bar{b}_{n} e^{2 u_{n}\left(\eta_{n}\right)}-\left(\frac{2 g_{n}}{\omega}-\bar{d}_{n}\right) e^{-\left(\bar{c}_{n, n-1} \omega+G_{n}\right)} e^{u_{n}\left(\eta_{n}\right)}+\bar{h}_{n} e^{-\left(\bar{c}_{n, n-1} \omega+G_{n}\right)}>0
$$

which implies that

$$
\ln B_{n}^{+}<u_{n}\left(\eta_{n}\right) \quad \text { or } \quad u_{n}\left(\eta_{n}\right)<\ln B_{n}^{-}
$$

In view of (3.34)-(3.63) and Lemma 3.3, we have

$$
\min \left\{\ln A_{i}^{+}, \ln B_{i}^{+}\right\}<u_{i}\left(\eta_{i}\right)<u_{i}\left(\xi_{i}\right)<\ln l_{i}^{+} \quad(1 \leq i \leq n)
$$


or

$$
\ln H_{i}<u_{i}\left(\eta_{i}\right)<u_{i}\left(\xi_{i}\right)<\max \left\{\ln A_{i}^{-}, \ln B_{i}^{-}\right\} \quad(1 \leq i \leq n),
$$

which imply that, for all $t \in \mathbb{T}$,

$$
\min \left\{\ln A_{i}^{+}, \ln B_{i}^{+}\right\}<u_{i}(t)<\ln l_{i}^{+} \quad \text { or } \quad \ln H_{i}^{-}<u_{i}(t)<\max \left\{\ln A_{i}^{-}, \ln B_{i}^{-}\right\} .
$$

For convenience, we denote

$$
G_{i}=\left(\ln H_{i}^{-}, \max \left\{\ln A_{i}^{-}, \ln B_{i}^{-}\right\}\right), \quad E_{i}=\left(\min \left\{\ln A_{i}^{+}, \ln B_{i}^{+}\right\}, \ln l_{i}^{+}\right) \quad(1 \leq i \leq n) .
$$

Clearly, $l_{i}^{ \pm}(i=1,2, \ldots, n), A_{i}^{ \pm}(i=1,2, \ldots, n)$, and $B_{i}^{ \pm}(i=1,2, \ldots, n)$ are independent of $\lambda$. For each $i=1,2, \ldots, n$, we choose an interval between two intervals $G_{i}$ and $E_{i}$ and denote it as $\Delta_{i}$, then define the set

$$
\left\{u=\left(u_{1}, u_{2}, \ldots, u_{n}\right)^{T} \in X: u_{i}(t) \in \Delta_{i}, t \in \mathbb{T}, i=1,2, \ldots, n\right\}
$$

Obviously, the number of the above sets is $2^{n}$. We denote these sets as $\Omega_{k}, k=1,2, \ldots, 2^{n}$. $\Omega_{k}$, $k=1,2, \ldots, 2^{n}$ are bounded open subsets of $X, \Omega_{i} \cap \Omega_{j}=\phi, i \neq j$. Thus $\Omega_{k}\left(k=1,2, \ldots, 2^{n}\right)$ satisfies the requirement (a) in Lemma 3.1.

Now we show that (b) of Lemma 3.1 holds; that is, we prove when $u \in \partial \Omega_{k} \cap \operatorname{Ker} L=$ $\partial \Omega_{k} \cap \mathbb{R}^{n}, Q N(u, 0) \neq(0,0, \ldots, 0)^{T}, k=1,2, \ldots, 2^{n}$. If it is not true, then when $u \in \partial \Omega_{k} \cap \operatorname{Ker} L=$ $\partial \Omega_{k} \cap \mathbb{R}^{n}, i=1,2, \ldots, 2^{n}$, constant vector $u=\left(u_{1}, u_{2}, \ldots, u_{n}\right)^{T}$ with $u \in \partial \Omega_{k}, k=1,2, \ldots, 2^{n}$, satisfies

$$
\begin{aligned}
-2 g_{1} & =\int_{\kappa}^{\kappa+\omega}\left\{a_{1}(t)-b_{1}(t) e^{u_{1}}-h_{1}(t) e^{-u_{1}}\right\} \Delta t, \\
& \vdots \\
-2 g_{i} & =\int_{\kappa}^{\kappa+\omega}\left\{-b_{i}(t) e^{u_{i}}+c_{i, i-1}(t) e^{u_{i-1}}-h_{i}(t) e^{-u_{i}}\right\} \Delta t, \quad 2 \leq i \leq n-1, \\
& \vdots \\
-2 g_{n} & =\int_{\kappa}^{\kappa+\omega}\left\{-b_{n}(t) e^{u_{n}}+c_{n, n-1}(t) e^{u_{n-1}}-h_{n}(t) e^{-u_{n}}\right\} \Delta t,
\end{aligned}
$$


that is,

$$
\begin{aligned}
-\frac{2 g_{1}}{\omega} & =\bar{a}_{1}-\bar{b}_{1} e^{u_{1}}-\bar{h}_{1} e^{-u_{1}}, \\
& \vdots \\
-\frac{2 g_{i}}{\omega} & =-\bar{b}_{i} e^{u_{i}}+\bar{c}_{i, i-1} e^{u_{i-1}}-\bar{h}_{i} e^{-u_{i}}, \quad 2 \leq i \leq n-1, \\
& \vdots \\
-\frac{2 g_{n}}{\omega} & =-\bar{b}_{n} e^{u_{n}}+\bar{c}_{n, n-1} e^{u_{n-1}}-\bar{h}_{n} e^{-u_{n}} .
\end{aligned}
$$

Similar to the process of (3.29)-(3.66), we obtain

$$
\ln H_{i}<u_{i}^{-}<\max \left\{\ln A_{i}^{-}, \ln B_{i}^{-}\right\}<\min \left\{\ln A_{i}^{+}, \ln B_{i}^{+}\right\}<u_{i}^{+}<\ln l_{i}^{+} \quad(1 \leq i \leq n) .
$$

Then $u$ belongs to one of $\Omega_{k} \cap \mathbb{R}^{n}, k=1,2, \ldots, 2^{n}$. This contradicts the fact that $u \in \partial \Omega_{k} \cap \mathbb{R}^{n}$, $k=1,2, \ldots, 2^{n}$. This proves that (b) in Lemma 3.1 holds.

Finally, in order to show that (c) in Lemma 3.1 holds, we only prove that for $u \in \partial \Omega_{k} \cap \operatorname{Ker} L=\partial \Omega_{k} \cap \mathbb{R}^{n}, k=1,2, \ldots, 2^{n}$, then it holds that $\operatorname{deg}\left\{\operatorname{JQN}(u, 0), \Omega_{k} \cap\right.$ $\left.\operatorname{Ker} L,(0,0, \ldots, 0)^{T}\right\} \neq 0$. To this end, we define the mapping $\phi:$ Dom $L \times[0,1] \rightarrow X$ by

$$
\phi(u, \mu)=\mu Q N(u, 0)+(1-\mu) G(u),
$$

here $\mu \in[0,1]$ is a parameter and $G(u)$ is defined by

$$
\left.G(u)=\left(\left(\begin{array}{c}
\frac{1}{\omega} \int_{\kappa}^{\kappa+\omega}\left(a_{1}(s)-b_{1}(s) e^{u_{1}(s)}-h_{1}(s) e^{-u_{1}(s)}\right) \Delta s+\frac{2 g_{1}}{\omega} \\
\vdots \\
\frac{1}{\omega} \int_{\kappa}^{\kappa+\omega}\left(c_{i, i-1}(s) l_{i-1}^{+}-b_{i}(s) e^{u_{i}(s)}-h_{i}(s) e^{-u_{i}(s)}\right) \Delta s+\frac{2 g_{i}}{\omega} \\
\vdots \\
\frac{1}{\omega} \int_{\kappa}^{\kappa+\omega}\left(c_{n, n-1}(s) l_{n-1}^{+}-b_{n}(s) e^{u_{n}(s)}-h_{n}(s) e^{-u_{n}(s)}\right) \Delta s+\frac{2 g_{n}}{\omega}
\end{array}\right),\left(\begin{array}{c}
0 \\
\vdots \\
0 \\
\vdots \\
0
\end{array}\right)\right\}_{k=1}^{q}\right),
$$


where $i=2,3, \ldots, n-1$. We show that for $u \in \partial \Omega_{k} \cap \operatorname{Ker} L=\partial \Omega_{k} \cap \mathbb{R}^{n}, k=1,2, \ldots, 2^{n}$, $\mu \in[0,1]$, then it holds that $\phi(u, \mu) \neq(0,0, \ldots, 0)^{T}$. Otherwise, parameter $\mu$ and constant vector $u=\left(u_{1}, u_{2}, \ldots, u_{n}\right)^{T} \in \mathbb{R}^{n}$ satisfy $\phi(u, \mu)=(0,0 \ldots, 0)^{T}$, that is,

$$
\begin{aligned}
0= & \mu \int_{\kappa}^{\kappa+\omega}\left[a_{1}(s)-b_{1}(s) e^{u_{1}}-h_{1}(s) e^{-u_{1}}+\frac{2 g_{1}}{\omega}\right] \Delta s \\
& +(1-\mu) \int_{\kappa}^{\kappa+\omega}\left[a_{1}(s)-b_{1}(s) e^{u_{1}}-h_{1}(s) e^{-u_{1}}+\frac{2 g_{1}}{\omega}\right] \Delta s, \\
\vdots & \\
0= & \mu \int_{\kappa}^{\kappa+\omega}\left[c_{i, i-1}(s) e^{u_{i-1}}-b_{i}(s) e^{u_{i}}-h_{i}(s) e^{-u_{i}}+\frac{2 g_{i}}{\omega}\right] \Delta s \\
& +(1-\mu) \int_{\kappa}^{\kappa+\omega}\left[c_{i, i-1}(s) l_{i-1}^{+}-b_{i}(s) e^{u_{i}}-h_{i}(s) e^{-u_{i}}+\frac{2 g_{i}}{\omega}\right] \Delta s, \\
\vdots & \mu \int_{\kappa}^{\kappa+\omega}\left[c_{n, n-1}(s) e^{u_{n-1}}-b_{n}(s) e^{u_{n}}-h_{n}(s) e^{-u_{n}}+\frac{2 g_{n}}{\omega}\right] \Delta s \\
& +(1-\mu) \int_{\kappa}^{\kappa+\omega}\left[c_{n, n-1}(s) l_{n-1}^{+}-b_{n}(s) e^{u_{n}}-h_{n}(s) e^{-u_{n}}+\frac{2 g_{n}}{\omega}\right] \Delta s,
\end{aligned}
$$

where $i=2,3, \ldots, n-1$, that is,

$$
\begin{gathered}
\bar{a}_{1}-\bar{b}_{1} e^{u_{1}}-\bar{h}_{1} e^{-u_{1}}=-\frac{2 g_{1}}{\omega}, \\
\vdots \\
\bar{c}_{i, i-1} l_{i-1}^{+}-\bar{b}_{i} e^{u_{i}}-\bar{h}_{i} e^{-u_{i}}=\mu\left(\bar{c}_{i, i-1} l_{i-1}^{+}-\bar{c}_{i, i-1} e^{u_{i-1}}\right)-\frac{2 g_{i}}{\omega}, \\
\vdots \\
\bar{c}_{n, n-1} l_{n-1}^{+}-\bar{b}_{n} e^{u_{n}}-\bar{h}_{n} e^{-u_{n}}=\mu\left(\bar{c}_{n, n-1} l_{n-1}^{+}-\bar{c}_{i, n-1} e^{u_{n-1}}\right)-\frac{2 g_{n}}{\omega},
\end{gathered}
$$

where $i=2,3, \ldots, n-1$. Following the argument of (3.29)-(3.66), we obtain

$$
\ln H_{i}<u_{i}^{-}<\max \left\{\ln A_{i}^{-}, \ln B_{i}^{-}\right\}<\min \left\{\ln A_{i}^{+}, \ln B_{i}^{+}\right\}<u_{i}^{+}<\ln l_{i}^{+} \quad(1 \leq i \leq n) .
$$


Equation (3.76) gives that $u$ belongs to one of $\Omega_{k} \cap \mathbb{R}^{n}, k=1,2, \ldots, 2^{n}$. This contradicts the fact that $u \in \partial \Omega_{k} \cap \mathbb{R}^{n}, k=1,2, \ldots, 2^{n}$. This proves $\phi(u, \mu) \neq(0,0, \ldots, 0)^{T}$ holds. Note that the system of algebraic equations

$$
\begin{gathered}
\bar{a}_{1}-\bar{b}_{1} e^{x_{1}}-\bar{h}_{1} e^{-x_{1}}=-\frac{2 g_{1}}{\omega}, \\
\vdots \\
\bar{c}_{i, i-1} l_{i-1}^{+}-\bar{b}_{i} e^{x_{i}}-\bar{h}_{i} e^{-x_{i}}=-\frac{2 g_{i}}{\omega}, \\
\vdots \\
\bar{c}_{n, n-1} l_{n-1}^{+}-\bar{b}_{n} e^{x_{n}}-\bar{h}_{n} e^{-x_{n}}=-\frac{2 g_{n}}{\omega},
\end{gathered}
$$

has $2^{n}$ distinct solutions since $\left(H_{1}\right),\left(H_{2}\right)$, and $\left(H_{3}\right)$ hold, $\left(x_{1}^{*}, x_{2}^{*}, \ldots, x_{n}^{*}\right)=\left(\ln \widehat{x}_{1}, \ln \widehat{x}_{2}\right.$,

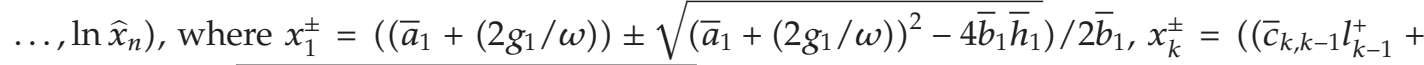

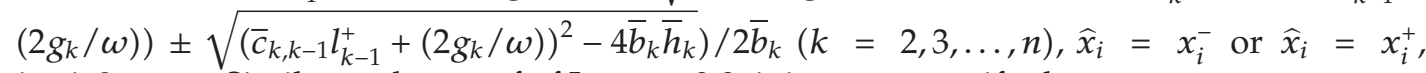
$i=1,2, \ldots, n$. Similar to the proof of Lemma 3.3, it is easy to verify that

$$
\ln H_{i}^{-}<\ln x_{i}^{-}<\max \left\{\ln B_{i}^{-}, \ln A_{i}^{-}\right\}<\min \left\{\ln A_{i}^{+}, \ln B_{i}^{+}\right\}<\ln x_{i}^{+}<\ln l_{i}^{+}, \quad i=1,2, \ldots, n .
$$

Therefore, $\left(x_{1}^{*}, x_{2}^{*}, \ldots, x_{n}^{*}\right)$ uniquely belongs to the corresponding $\Omega_{k}$. Since Ker $L=$ $\operatorname{Im} Q$, we can take $J=I$. A direct computation gives, for $k=1,2, \ldots, 2^{n}$,

$$
\begin{aligned}
\operatorname{deg}\left\{\operatorname{JQN}(u, 0), \Omega_{k} \cap \operatorname{Ker} L,(0,0, \ldots, 0)^{T}\right\} & =\operatorname{deg}\left\{\phi(u, 1), \Omega_{k} \cap \operatorname{Ker} L,(0,0, \ldots, 0)^{T}\right\} \\
& =\operatorname{deg}\left\{\phi(u, 0), \Omega_{k} \cap \operatorname{Ker} L,(0,0, \ldots, 0)^{T}\right\} \\
& =\operatorname{sign}\left[\prod_{i=1}^{n}\left(-\bar{b}_{i} x_{i}^{*}+\frac{\bar{h}_{i}}{x_{i}^{*}}\right)\right] .
\end{aligned}
$$

Since $\left(\bar{a}_{1}+\left(2 g_{1} / \omega\right)\right)-\bar{b}_{1} x_{1}^{*}-\left(\bar{h}_{1} / x_{1}^{*}\right)=0,\left(\bar{c}_{i, i-1} l_{i-1}^{+}+\left(2 g_{i} / \omega\right)\right)-\bar{b}_{i} x_{i}^{*}-\left(\bar{h}_{i} / x_{i}^{*}\right)=0(i=2,3, \ldots, n)$, then

$$
\begin{aligned}
\operatorname{deg} & \left\{\operatorname{JQN}(u, 0), \Omega_{k} \cap \operatorname{Ker} L,(0,0, \ldots, 0)^{T}\right\} \\
& =\operatorname{sign}\left[\prod_{i=2}^{n}\left(\bar{a}_{1}+\frac{2 g_{1}}{\omega}-2 \bar{b}_{1} x_{1}^{*}\right)\left(\bar{c}_{i, i-1} l_{i-1}^{+}+\frac{2 g_{i}}{\omega}-2 \bar{b}_{i} x_{i}^{*}\right)\right]= \pm 1, \quad k=1,2, \ldots, 2^{n} .
\end{aligned}
$$

So far, we have proved that $\Omega_{k}\left(k=1,2, \ldots, 2^{n}\right)$ satisfies all the assumptions in Lemma 3.1. Hence, system (1.3) has at least $2^{n}$ different $\omega$-periodic solutions. This completes the proof of Theorem 3.5. 
If $n=2$, then (1.3) is changed into the predator-prey system (3.81). The following Corollary 3.6 gives the existence of multiple periodic solutions for system (3.81).

Corollary 3.6. For the following predator-prey system on $\omega$-periodic time scales $\mathbb{T}$,

$$
\begin{aligned}
u_{1}^{\Delta}(t)= & a_{1}(t)-b_{1}(t) e^{u_{1}\left(t-\tau_{11}(t)\right)}-c_{12}(t) e^{u_{2}\left(t-\tau_{12}(t)\right)}-h_{1}(t) e^{-u_{1} t}, \quad t \neq t_{k}, \\
u_{2}^{\Delta}(t)= & -d_{2}(t)-b_{2}(t) e^{u_{2}\left(t-\tau_{22}(t)\right)}+c_{21}(t) e^{u_{1}\left(t-\tau_{21}(t)\right)}-h_{2}(t) e^{-u_{2}(t)}, \quad t \neq t_{k} \\
& \Delta u_{i}\left(t_{k}\right)=u_{i}\left(t_{k}^{+}\right)-u_{i}\left(t_{k}^{-}\right)=\ln \left(1+g_{i k}\right), \quad i=1,2, t=t_{k}
\end{aligned}
$$

if the inequalities $\left(\bar{a}_{1}+\left(2 g_{1} / \omega\right)-\bar{c}_{12} l_{2}^{+}\right) e^{-(1 / 2)\left(\bar{a}_{1} \omega+G_{1}\right)}>2 \sqrt{\bar{b}_{1} \bar{h}_{1}}$ and $\left(\left(2 g_{2} / \omega\right)-\bar{d}_{2}\right) e^{-(1 / 2)\left(\bar{c}_{21} \omega+G_{2}\right)}>$ $2 \sqrt{\bar{b}_{2} \bar{h}_{2}}$ hold, then system (3.81) at least exist four w-periodic solutions.

\section{Illustrative Examples}

Consider the following time-delay predator-prey system with impulses on time scales:

$$
\begin{gathered}
u_{1}^{\Delta}(t)=a_{1}(t)-b_{1}(t) e^{u_{1}\left(t-\tau_{11}(t)\right)}-c_{12}(t) e^{u_{2}\left(t-\tau_{12}(t)\right)}-h_{1}(t) e^{-u_{1}(t)}, \\
u_{2}^{\Delta}(t)=-d_{2}(t)-b_{2}(t) e^{u_{2}\left(t-\tau_{22}(t)\right)}+c_{21}(t) e^{u_{1}\left(t-\tau_{21}(t)\right)}-h_{2}(t) e^{-u_{2}(t)}, \\
\Delta x_{1}(1)=-2, \quad \Delta x_{1}(3)=2, \quad \Delta x_{2}(1)=-1, \quad \Delta x_{2}(3)=3,
\end{gathered}
$$

where $a_{1}(t)=(3+\sin (0.5 \pi t)) / 2, b_{1}(t)=(2+\sin (0.5 \pi t)) / 10 e^{4}, h_{1}(t)=(4+\cos (0.5 \pi t)) / 20 e^{4}$, $\tau_{11}(t)=|\sin (0.5 \pi t)|, \tau_{12}(t)=|\cos (0.5 \pi t)|, d_{2}(t)=(2+\cos (0.5 \pi t)) / 25, b_{2}(t)=(5+$ $\cos (0.5 \pi t)) / 10, h_{2}(t)=(2+\cos (0.5 \pi t)) / 25 e^{2}, c_{21}(t)=(2+\sin (\pi t)) / 8, \tau_{22}(t)=|\cos (\pi t)|$, $\tau_{21}(t)=|\sin (\pi t)|$.

If $\mathbb{T}=\mathbb{R}$, then (4.1) reduces to the following system:

$$
\begin{gathered}
\dot{u}_{1}(t)=a_{1}(t)-b_{1}(t) e^{u_{1}\left(t-\tau_{11}(t)\right)}-c_{12}(t) e^{u_{2}\left(t-\tau_{12}(t)\right)}-h_{1}(t) e^{-u_{1}(t)}, \\
\dot{u}_{2}(t)=-d_{2}(t)-b_{2}(t) e^{u_{2}\left(t-\tau_{22}(t)\right)}+c_{21}(t) e^{u_{1}\left(t-\tau_{21}(t)\right)}-h_{2}(t) e^{-u_{2}(t)}, \\
\Delta x_{1}(1)=-2, \quad \Delta x_{1}(3)=2, \quad \Delta x_{2}(1)=-1, \quad \Delta x_{2}(3)=3 .
\end{gathered}
$$

Let $x_{i}(t)=e^{u_{i}(t)}(i=1,2)$, then (4.2) can be changed into the following system:

$$
\begin{gathered}
\dot{x}_{1}(t)=x_{1}(t)\left(a_{1}(t)-b_{1}(t) x_{1}\left(t-\tau_{11}(t)\right)-c_{12}(t) x_{2}\left(t-\tau_{12}(t)\right)\right)-h_{1}(t), \\
\dot{x}_{2}(t)=x_{2}(t)\left(-d_{2}(t)-b_{2}(t) x_{2}\left(t-\tau_{22}(t)\right)+c_{21}(t) x_{1}\left(t-\tau_{21}(t)\right)\right)-h_{2}(t), \\
\Delta x_{1}(1)=e^{-2}-1, \quad \Delta x_{1}(3)=e^{2}-1, \quad \Delta x_{2}(1)=e^{-1}-1, \quad \Delta x_{2}(3)=e^{3}-1 .
\end{gathered}
$$


Since

$$
\begin{aligned}
l_{1}^{ \pm} & =\frac{\left(\bar{a}_{1}+\left(2 g_{1} / \omega\right)\right) e^{\bar{a}_{1} \omega+G_{1}} \pm \sqrt{\left(\left(\bar{a}_{1}+\left(2 g_{1} / \omega\right)\right) e^{\bar{a}_{1} \omega+G_{1}}\right)^{2}-4 \bar{b}_{1} \bar{h}_{1} e^{\bar{a}_{1} \omega+G_{1}}}}{2 \bar{b}_{1}} \\
& =\frac{\left(15 e^{8} \pm 3 \sqrt{225 e^{16}-16}\right)}{4 e^{-4}}, \\
l_{2}^{ \pm} & =\frac{\left(\bar{c}_{21}+\left(2 g_{2} / \omega\right)\right) e^{\bar{c}_{21} \omega+G_{2}} \pm \sqrt{\left(\left(\bar{c}_{21}+\left(2 g_{2} / \omega\right)\right) e^{\bar{c}_{21} \omega+G_{2}}\right)^{2}-4 \bar{b}_{2} \bar{h}_{2} e^{\bar{c}_{21} \omega+G_{2}}}}{2 \bar{b}_{2}} \\
& =\frac{15 e^{4} \pm \sqrt{225 e^{8}-64}}{20 e^{-2}} .
\end{aligned}
$$

Take $c_{12}(t)=c_{12}(t+2 \pi)>0$, such that $\bar{c}_{12}=1 / l_{2}^{+}$, then

$$
\begin{gathered}
\left(\bar{a}_{1}+\frac{2 g_{1}}{\omega}-\bar{c}_{12} l_{2}^{+}\right) e^{-(1 / 2)\left(\bar{a}_{1} \omega+G_{1}\right)}=\frac{1}{2} e^{-4}>\frac{2}{5} e^{-4}=2 \sqrt{\bar{b}_{1} \bar{h}_{1}}, \\
\left(\frac{2 g_{2}}{\omega}-\bar{d}_{2}\right) e^{-(1 / 2)\left(\bar{c}_{21} \omega+G_{2}\right)}=\frac{21}{25} e^{-2}>\frac{2}{5} e^{-2}=2 \sqrt{\bar{b}_{2} \bar{h}_{2}} .
\end{gathered}
$$

Therefore, all conditions of Corollary 3.6 are satisfied. By Corollary 3.6, system (4.1) has at least four 4-periodic solutions.

\section{Acknowledgments}

This work are supported by the National Natural Sciences Foundation of People's Republic of China under Grant (no. 11161025), Yunnan Province Natural Scientific Research Fund Project (no. 2011FZ058), and Yunnan Province Education Department Scientific Research Fund Project (no. 2011Z001).

\section{References}

[1] M. L. Rosenzweig, "Paradox of enrichment: destabilization of exploitation ecosystems in ecological time," Science, vol. 171, no. 3969, pp. 385-387, 1971.

[2] A. Hastings and T. Powell, "Chaos in a three-species food chain," Ecology, vol. 72, no. 3, pp. 896-903, 1991.

[3] K. McCann and P. Yodzis, "Biological conditions for chaos in a three-species food chain," Ecology, vol. 75, no. 2, pp. 561-564, 1994.

[4] P. A. Abrams and J. D. Roth, "The effects of enrichment of three-species food chains with nonlinear functional responses," Ecology, vol. 75, no. 4, pp. 1118-1130, 1994.

[5] Yu. A. Kuznetsov and S. Rinaldi, "Remarks on food chain dynamics," Mathematical Biosciences, vol. 134, no. 1, pp. 1-33, 1996.

[6] A. Gragnani, O. De Feo, and S. Rinaldi, "Food chains in the chemostat: relationships between mean yield and complex dynamics," Interim Report IR-97-042, International Institute for Applied Systems Analysis, Laxenburg, Austria, 1997.

[7] C.-H. Chiu and S.-B. Hsu, "Extinction of top-predator in a three-level food-chain model," Journal of Mathematical Biology, vol. 37, no. 4, pp. 372-380, 1998. 
[8] Z. Wen and C. Zhong, "Non-constant positive steady states for the HP food chain system with crossdiffusions," Mathematical and Computer Modelling, vol. 51, no. 9-10, pp. 1026-1036, 2010.

[9] G.-H. Cui and X.-P. Yan, "Stability and bifurcation analysis on a three-species food chain system with two delays," Communications in Nonlinear Science and Numerical Simulation, vol. 16, no. 9, pp. 3704 3720, 2011.

[10] Y. Li and K. Zhao, "Existence of $2^{n}$ positive periodic solutions to n-species nonautonomous food chains with Harvesting terms," Advances in Difference Equations, vol. 2010, Article ID 262461, 17 pages, 2010.

[11] D. BaĬnov and P. Simeonov, Impulsive Differential Equations: Periodic Solutions and Applications, Pitman Monographs and Surveys in Pure and Applied Mathematics, Longman Scientific \& Technical, Harlow, UK, 1993.

[12] V. Lakshmikantham, D. D. Bar̆nov, and P. S. Simeonov, Theory of Impulsive Differential Equations, vol. 6, World Scientific Publishing, Teaneck, NJ, USA, 1989.

[13] J. G. P. Gamarra and R. V. Solé, "Complex discrete dynamics from simple continuous population models," Bulletin of Mathematical Biology, vol. 64, no. 3, pp. 611-620, 2002.

[14] M. Bohner and A. Peterson, Dynamic Equations on Time Scales: An Introduction with Applications, Birkhäauser, Boston, Mass, USA, 2001.

[15] M. Bohner and A. Peterson, Advances in Dynamic Equations on Time Scales, Birkhäauser, Boston, Mass, USA, 2003.

[16] S. Hilger, "Analysis on measure chains-a unified approach to continuous and discrete calculus," Results in Mathematics. Resultate der Mathematik, vol. 18, no. 1-2, pp. 18-56, 1990.

[17] R. Gaines and J. Mawhin, Coincidence Degree and Nonlinear Differetial Equitions, Springer, Berlin, Germany, 1977.

[18] Y. Chen, "Multiple periodic solutions of delayed predator-prey systems with type IV functional responses," Nonlinear Analysis: Real World Applications, vol. 5, no. 1, pp. 45-53, 2004.

[19] Q. Wang, B. Dai, and Y. Chen, "Multiple periodic solutions of an impulsive predator-prey model with Holling-type IV functional response," Mathematical and Computer Modelling, vol. 49, no. 9-10, pp. 18291836, 2009.

[20] D. Hu and Z. Zhang, "Four positive periodic solutions to a Lotka-Volterra cooperative system with harvesting terms," Nonlinear Analysis: Real World Applications, vol. 11, no. 2, pp. 1115-1121, 2010.

[21] Z. Zhang and T. Tian, "Multiple positive periodic solutions for a generalized predator-prey system with exploited terms," Nonlinear Analysis: Real World Applications, vol. 9, no. 1, pp. 26-39, 2008.

[22] K. Zhao and Y. Ye, "Four positive periodic solutions to a periodic Lotka-Volterra predatory-prey system with harvesting terms," Nonlinear Analysis: Real World Applications, vol. 11, no. 4, pp. 24482455, 2010.

[23] K. Zhao and Y. Li, "Four positive periodic solutions to two species parasitical system with harvesting terms," Computers $\mathcal{E}$ Mathematics with Applications, vol. 59, no. 8, pp. 2703-2710, 2010.

[24] Y. Li, K. Zhao, and Y. Ye, "Multiple positive periodic solutions of $n$ species delay competition systems with harvesting terms," Nonlinear Analysis: Real World Applications, vol. 12, no. 2, pp. 1013-1022, 2011.

[25] H. Fang, "Existence of at least two periodic solutions for a competition system of plankton allelopathy on time scales," Journal of Applied Mathematics, vol. 2012, Article ID 602679, 14 pages, 2012.

[26] Y. Xing, M. Han, and G. Zheng, "Initial value problem for first-order integro-differential equation of Volterra type on time scales," Nonlinear Analysis: Theory, Methods \& Applications, vol. 60, no. 3, pp. 429-442, 2005. 


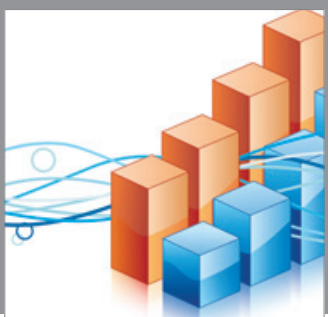

Advances in

Operations Research

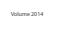

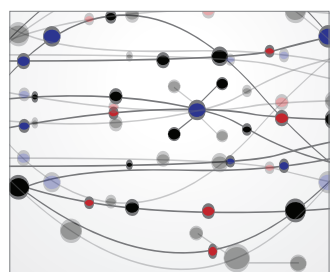

\section{The Scientific} World Journal
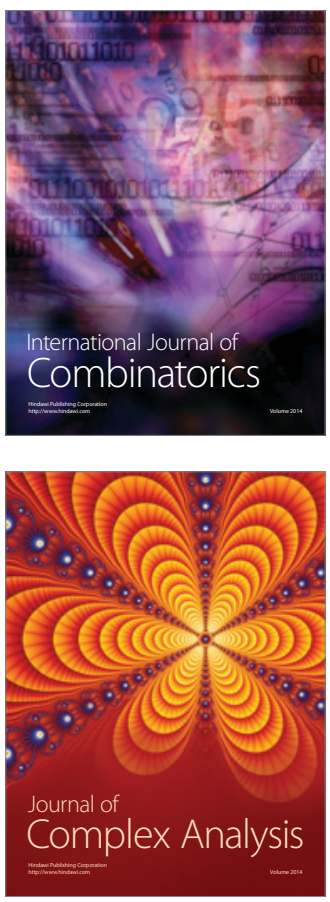

International Journal of

Mathematics and

Mathematical

Sciences
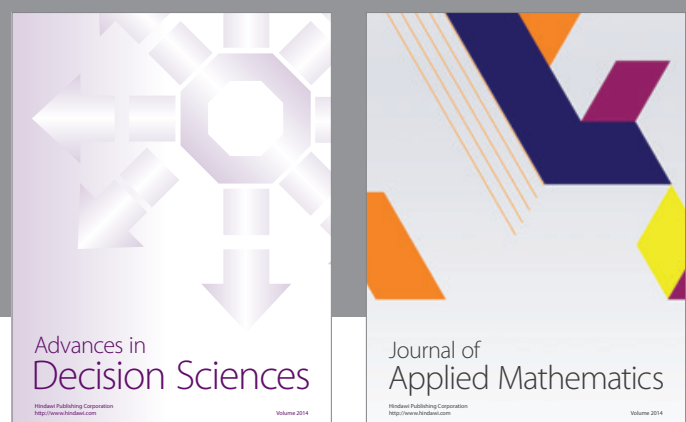

Journal of

Applied Mathematics
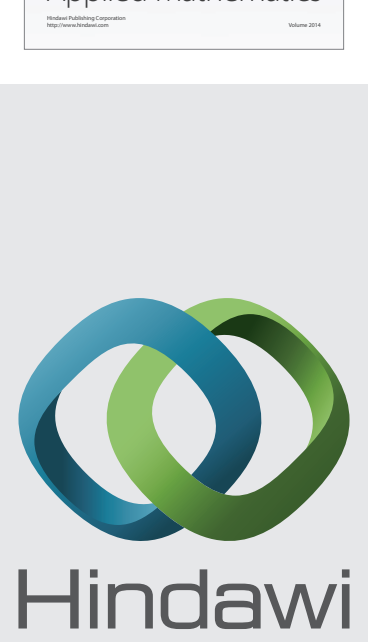

Submit your manuscripts at http://www.hindawi.com
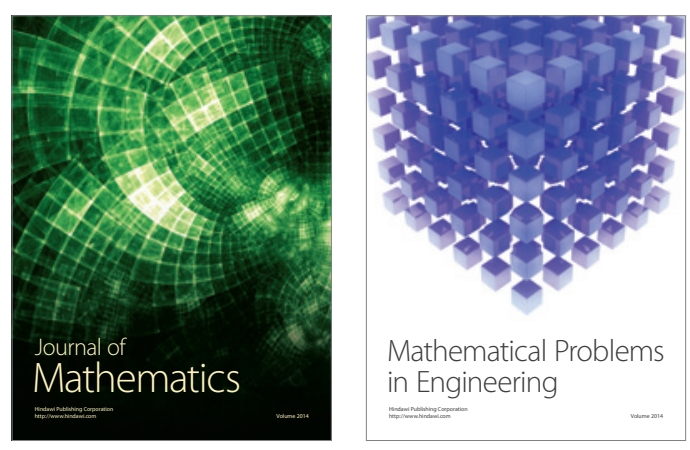

Mathematical Problems in Engineering
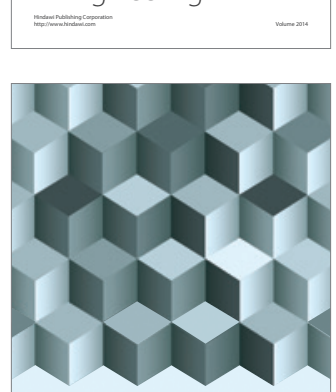

Journal of

Function Spaces
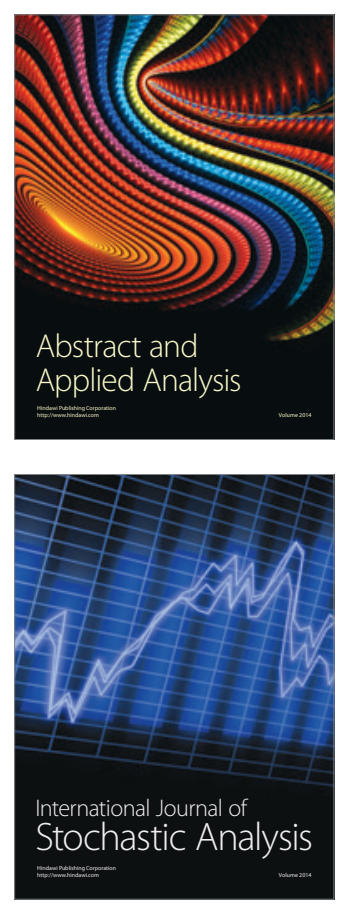

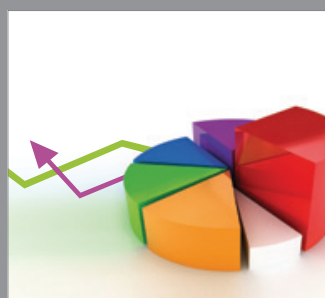

ournal of

Probability and Statistics

Promensencen
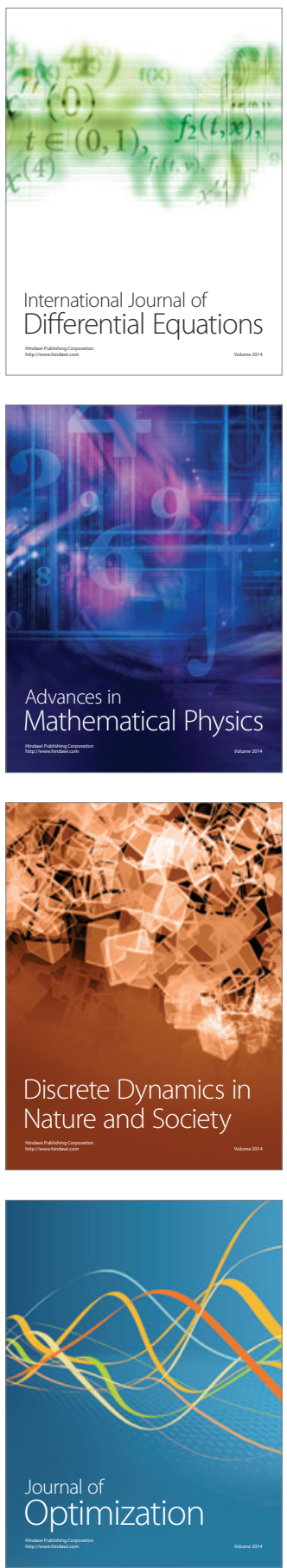